\title{
Unemployment insurance design: Inducing moving and retraining is
}

\author{
John Hassler ${ }^{\mathrm{a}, *}$, José V. Rodríguez Mora ${ }^{\mathrm{b}}$ \\ anstitute for International Economic Studies, Stockholm University, S-106 91 Stockholm, \\ Sweden, IZA, CEPR and CESIfo \\ ${ }^{\mathrm{b}}$ University of Edinburgh, Universitat Pompeu Fabra, CREA and CEPR
}

Received 8 September 2006; accepted 14 August 2007

Available online 6 September 2007

\begin{abstract}
Evidence suggests that unemployed individuals can affect their job prospects by undertaking a costly action like deciding to move or retrain. Realistically, such an opportunity only arises for some individuals and the identity of those may be unobservable ex ante. The problem of characterizing constrained optimal unemployment insurance in this case has been neglected in previous literature. We construct a model of optimal unemployment insurance where multiple incentive constraints are easily handled. The model is used to analyze the case when an incentive constraint involving moving costs must be respected in addition to the standard constraint involving costly unobservable jobsearch. Absent wealth effects on behavior, we derive closed-form solutions showing that when the moving/retraining incentive constraint binds, unemployment benefits should increase over the unemployment spell, with an initial period with low benefits and an increase after this period has expired.
\end{abstract}

(C) 2007 Elsevier B.V. All rights reserved.

JEL classification: J65; J64; E24

Keywords: Unemployment benefits; Search; Moral hazard; Adverse selection

\footnotetext{
This is a substantial revision of a previous version that circulated under the title "Should UI benefits really fall over time?".

${ }^{*}$ Corresponding author. Tel.: +468162070 ; fax: +468161443 .

E-mail addresses: John@Hassler.se (J. Hassler), sevimora@gmail.com (J.V. Rodríguez Mora).
} 


\section{Introduction}

An important feature of the modern welfare state is the existence of an extensive unemployment insurance (UI) system. It is now well established that the design of the unemployment insurance affects the incidence of unemployment by distorting the incentives of unemployed to search for a job (see, e.g., Holmlund, 1998 for a survey). This has motivated a growing literature on how the UI system should be designed to make an optimal trade-off between providing good insurance, on the one hand, and not distorting the incentives too much, on the other. The key informational friction in this literature is that search activity cannot be monitored, so sufficient search incentives must be provided.

The contribution of this paper is twofold. The first contribution is to focus on an important informational friction that has been largely neglected in the literature. We will consider the case when individuals who become unemployed have different opportunities to find a new job. However, we assume that the insurer cannot (perfectly) observe these differences. Specifically, we assume that some, but not all, unemployed can increase the probability of being hired by undertaking a costly investment, e.g., by retraining or moving to a location with better employment prospects. Under the realistic assumption that the insurer is unable to observe who has this option, an incentive problem arises and failure to take this into account may lead to sub-optimal UI-design. One direct way of mitigating the problem would be to offer subsidies to moving or retraining. While we will discuss this case at the end of the paper, our main case is when full cost-compensation is not feasible, for example because the insurer cannot fully distinguish voluntary and involuntary jobseparations.

Although an empirical investigation is outside the scope of this paper, we argue that the consequences of not providing reasonable incentives for people to move or retrain may be of substantial quantitative importance. For instance, Bartel (1979) documents that the proportion of geographical mobility in the U.S. caused by the decision to change jobs is one-half of all migration decisions for young workers and one third of all migration decisions for workers aged above 45. Furthermore, geographical mobility is substantially lower in continental Europe, and Hassler et al. (2005) document in panel-data a negative correlation between geographical mobility and UI-generosity as well as between mobility and aggregate unemployment rates. Other empirical documentations of the link between unemployment and geographical mobility are DaVanzo (1978), Pissarides and Wadsworth (1989) and McCormick (1997).

The second contribution of our paper is more methodological. Search incentives and incentives to move are generally not independent and should therefore be jointly analyzed. The reason why moving incentives are not included in the standard analysis is that multiple incentive constraints with different characteristics are difficult to analyze. Including both search and moving/retraining incentive constraints complicates the analysis, since it is difficult to evaluate which of many constraints are binding, in particular when unemployment benefits are allowed to be non-constant. Suppose, for example, that the benefit schedule contains $x$ tiers, so that the benefit level $b$ is an element of $B \equiv\left\{b_{1}, b_{2}, \ldots, b_{x}\right\}$. The incentive constraint for an individual at a particular tier then depends on benefits in all tiers that the individual could eventually end up, in general all elements of $B$. The methodological contribution of the paper is to show that the problem of finding the optimal benefit structure can be formulated in such a way that all incentive 
constraints are linear and parallel or independent of each other. It is then immediate to check which constraints are binding and optimal benefits can easily be characterized, both graphically and analytically. We will provide analytical expressions for the (constrained) optimal benefit schedule and, in particular, focus on the issue of whether benefits should increase or decrease over time. Our model easily lends itself to allowing multiple incentive problems and adding, for example, a moral hazard problem in job-retention effort as in Wang and Williamson (1996) should be straightforward.

There exists an extensive literature on the optimal design of social insurance schemes under moral hazard. In one line of research, the question is how to optimally set a time invariant benefit level in a two state setting (e.,g., employment and unemployment) where an individually costly and unobserved action (job search) determines the transition probability from one of the states. A seminal contribution is Baily (1978), who uses a two period model to derive a formula for the optimal benefit level that only depends on three parameters: the degree of risk aversion, the consumption-smoothing benefit of UI, and the elasticity of unemployment duration to the benefit rate. Chetty (2006) shows that a generalized formula, including also the degree of prudence, is applicable in a surprisingly more general and dynamic setting, provided the focus is on time invariant benefits and two states. Given these results, empirical analysis on the sensitivity of consumption and unemployment duration to the benefit level, like for example David Card and Weber (2007), can then be used to "calibrate" the formula for the optimal benefit level and no direct evidence on, for example, the ability the individual has to self-insure, is needed. The generality of this approach of course comes at a cost - it is not enough to consider the change in consumption as unemployment is entered and how this is affected by changes in UI. Rather, it is the sensitivity of lifetime average total consumption to UI benefits that must be estimated. However, Shimer and Werning(2007) recently show that the reservation wage of individuals can be used as an alternative summary measure of worker utility. Arguably, this is easier to measure since it can be observed without access to panel data. Our work is closely related to this line of research in the sense that a key variable of focus is how much the individual decides to change her consumption when her labor market status changes. In principle, the previous analysis could allow for a moving choice that affects the duration of unemployment. We add to this by allowing heterogeneity among unemployed and show that this has implications for the optimal time profile of benefits. Empirical work on how the time profile of benefits affects unemployment duration and consumption should therefore be valuable.

By allowing time varying benefits and our work is related to the line of papers following the influential papers by Shavell and Weiss (1979) and Hopenhayn and Nicolini (1997). Here, the focus is on the optimal time profile of benefits chosen by a planner who can control consumption of the individual but not her search intensity. A key result here is that the optimal trade-off between insurance and incentive provision implies that consumption should fall over time as long as the individual remains unemployed. A standard interpretation of this result is that unemployment benefits should fall over time. However, this interpretation relies on the assumption that the insurer can perfectly control individual consumption by determining the benefit levels. In a recent line of papers (e.g., Pavoni, 2006; Arpad and Pavoni, 2005; Werning, 2002; Shimer and Werning, 2005), the individual is allowed to make her own consumption decisions by allowing access to a perfect market for saving and borrowing. Then, as assets are run down during an unemployment spell, consumption falls over time by choice of the individual also with constant benefits. In fact, 
under constant absolute risk-aversion, there is no need to affect the rate of decline of consumption and a constant benefit level is optimal if the moral hazard problem is stationary (see Werning, 2002; Shimer and Werning, 2005).

The fact that our model easily and analytically can handle several incentive constraints hinges on the absence of wealth effects, which is due to some key assumptions. First, we follow the papers mentioned above by assuming access to a safe bond. Second, we assume constant absolute risk-aversion. ${ }^{1}$ The absence of wealth effects on incentives implies that we can induce people to voluntarily move or retrain, as well as to search for a job, using simple benefit schemes with a limited number of benefit levels that are independent of the full employment history of the agent. With decreasing absolute risk aversion or financial frictions, it could be the case that unemployed individuals do not retrain or move until they have run down their assets to some critical level and then decide to move. A similar case could arise if unemployed individuals learn about their prospects over time, starting their unemployment spell with optimistic beliefs and then turn more pessimistic. Clearly, this would not only complicate the analysis but could also alter our results regarding the optimal time-profile of benefits.

Regarding our assumption of access to a market for borrowing and saving, we want to stress that there is empirical evidence indicating that precautionary saving is used to selfinsure against unemployment risk. Using PSID, Gruber (1997) finds that, in the absence of UI, consumption falls by only $22 \%$ when an individual becomes unemployed, showing that individuals are able to smooth consumption also when there is no UI. Similarly, Engen and Gruber (2001) show that UI crowds out financial savings, indicating that households use financial markets to self-insure against unemployment risk. $^{2}$ It is nevertheless clear that neither of the key assumptions is perfectly realistic and a quantitative analysis might require wealth effects, either because of non-constant absolute risk aversion and/or because of variations in the bite of liquidity constraints. However, we hope that illustrating a mechanism not previously explored in the literature might provide guidance for future quantitative work. We return to this issue in the conclusion.

The paper is structured in the following way. The model is presented in Section 2, where the relevant value functions are derived in Section 2.1. The formal optimality problem is defined and solved in Section 3. In Section 3.1, we show the methodology in the simplest case with a constant benefit level and in Section 3.2, we allow time varying benefits. In Section 4, the optimal insurance scheme is characterized under different assumptions on search and moving costs. Section 5 relaxes some of the assumptions in the previous section and Section 6 concludes. Some proofs are given in the main text, others in the appendix and the remaining ones are available from the authors upon request.

\section{The model}

Consider an economy in continuous time where individuals can either be employed or unemployed. They have access to a market for safe saving and borrowing with an

\footnotetext{
${ }^{1}$ Other frictions, like irreversibilities in durables consumption and other consumption commitments, would also generically make incentive constraints wealth dependent. Recently, Chetty and Szeidl (2007) show that consumption commitments may affect risk preferences and their wealth dependence.

${ }^{2}$ Also if access to the formal capital market is limited, alternative means of smoothing consumption may exist, see e.g., Cullen and Gruber (2000).
} 
exogenous return $r$, equal to the subjective discount rate (possibly including a positive probability of dying). Unemployed individuals can affect their chances of finding a job. As noted in the introduction, we will focus on the case when some, but not necessarily all, unemployed individuals can make a costly investment increasing their chances of becoming employed. Allowing unobservable heterogeneity in this respect creates an informational problem similar to an adverse selection problem. ${ }^{3}$ In addition, we will allow the more standard moral hazard problem where search activity entails a flow cost.

Specifically, we assume that an employed individual, who is said to be in state 1 , loses her job at the exogenous rate $q$. With probability $p \in[0,1]$ those who loose their job can undertake a costly investment. We will interpret this as representing a cost of moving, denoted $m>0$ (for example between geographical locations or between occupations requiring some retraining). For simplicity, we assume that the opportunity to undertake this investment arises immediately upon separation and if the unemployed pays this cost ("moves"), she is immediately rehired. In Section 5.1, we relax this assumption by assuming that moving opportunities arise with a finite arrival rate.

Unemployed who cannot move or decide not to move and who search for a job find one at an exogenous rate $h$. Searching has a cost of $s \geqslant 0$ per unit of time. In Section 5.2, we instead assume that unemployed individuals can choose a continuous hiring rate at an increasing and convex cost $s(h)$. We may consider search costs as representing the opportunity cost of searching, arising from, for example, some alternative valuable activity. Whether the agent actually searches or not and whether she has the opportunity to move are assumed to be her own private information. To make the problem interesting, we assume that it is socially optimal to induce individuals to search and move (if they have the opportunity). ${ }^{4}$ It is easily shown that under this assumption, agents with the option of moving should be induced to do so immediately. Therefore, in the optimal solution of the baseline model, no mass of agents should be unemployed while having the opportunity to move. In Section 5.1, on the other hand, unemployed individuals search for both job and moving opportunities.

A key question we want to analyze is if and how UI benefits should change over the duration of the unemployment spell. To answer this question, we make two assumptions that will simplify the analysis and make graphical representations of our results possible. First, we assume the benefit schedule to be a ladder with a finite number of steps. In fact, we only allow two benefit levels, $b_{2}$ and $b_{3}$, but the extension to any a finite number of benefit levels is straightforward. Moreover, we can show that our main results would not change by allowing more than two benefit tiers - with $x$ benefit tiers, only the first should have a unique value, all latter benefit tiers should be identical. ${ }^{5}$ Second, we assume transition between the steps in the benefit schedule to occur with a constant hazard rate $f$. Individuals who lose their jobs enter state 2 and receive benefits $b_{2}$. In state 2 , they face a constant hazard rate $f$ of entering state 3 and then receiving benefits $b_{3}{ }^{6}$ Motivated by

\footnotetext{
${ }^{3}$ There are very few papers on UI which deal with adverse selection. One recent paper is Hagedorn et al. (2003), where individuals with different hiring rates are separated by being offered different "benefit menus".

${ }^{4}$ We return to this assumption in Section 5.1.

${ }^{5}$ Proof available upon request.

${ }^{6}$ This assumption implies that search incentives remain constant as long as the individual remains in state 2 . An alternative would be to use discrete time and assume that short-term UI benefits are paid for one period only, as done by e.g., Cahuc and Lehmann (2000). Assuming that UI benefits change after some fixed period of time would make search incentives depend on the remaining time of current benefits and considerably complicate the analysis with little gain.
} 
real-world practical considerations, and in contrast to, e.g., Hopenhayn and Nicolini (1997), we assume that benefit levels can only be given conditional on current unemployment status ( 2 or 3 ), not conditional on employment history or asset holdings. ${ }^{7}$

Given the multiple incentive constraints, an extended unemployment insurance, where individuals can choose between different menus, may be better than a simple two-tier system. In Section 5.4, we allow such a scheme, showing that our results regarding under which conditions UI benefits should be increasing and when they should be decreasing remain valid in the case of menu-based insurance.

The simplest and most obvious way of interpreting the unemployment states is as an indication of the passage of time: individuals in state 3 have, on average, been unemployed longer than individuals in state 2 . Therefore, we label state 2 as short-term unemployment and state 3 as long-term unemployment. Our preferred interpretation of the third state is that it is a purely administrative state and we may allow the insurance provider to choose $f$. In this case, it is natural to assume that search costs $(s)$ and hiring probability $(h)$ are the same in both states.

We may also interpret the third state as representing loss of skills during unemployment in the sense of job-finding rates and search costs developing disadvantageously over the unemployment spell. As an extension, we modify the model so that with a constant instantaneous probability $f$, unemployed individuals suffer a shock, and their search costs increase $\left(s_{2}<s_{3}\right)$ and/or their hiring probabilities decrease $\left(h_{2}>h_{3}\right)$. Although this interpretation raises issues about observability, we abstain from these and assume benefits to be paid contingent on whether the individual is in state 2 or 3 .

Individuals maximize their intertemporal utility, given by

$$
E \int_{0}^{\infty} \mathrm{e}^{-r t} U\left(c_{t}\right) \mathrm{d} t
$$

where $c_{t}$ is consumption at time $t$ and $r$ is the subjective discount rate. To facilitate analytical solutions when individuals have access to markets for saving and borrowing, we choose the CARA utility function

$$
U\left(c_{t}\right) \equiv-\mathrm{e}^{-\gamma c_{t}},
$$

where $\gamma$ is the coefficient of absolute risk aversion. All individuals are born (enter the labor market) as employed without assets and are identical at that point.

The purpose of this paper is to discuss how an unemployment insurance system should be constructed when there are incentive problems. To this end, we want to remove other motives for unemployment benefits than providing insurance. In particular, we are in this paper not interested in motives for using the UI system to create non-actuarial transfers between individuals with different characteristics. ${ }^{8}$ Therefore, we assume that individuals face an actuarially fair insurance. This means that when an individual enters the labor force, the expected present discounted value of the benefits she will receive during her lifetime exactly balances the expected present discounted value of her contributions. An alternative interpretation of actuarial fairness is that in a decentralized equilibrium, where individuals can sign binding insurance contracts with competitive insurance companies

\footnotetext{
${ }^{7}$ In fact, under CARA utility, also this assumption is innocuous.

${ }^{8}$ For positive implications, the redistributive elements of unemployment insurance are, however, likely to be central. See e.g., Wright (1986).
} 
when entering their first job, actuarial fairness is identical to a break-even condition for the insurance companies, which would be satisfied under perfect competition. ${ }^{9}$

Without loss of generality, we let individuals pay lump-sum taxes, denoted $\tau$, implying that

$$
\dot{A}_{t}=r A_{t}+y-c_{t}-\tau,
$$

except at the points in time when the cost of moving is paid, and where $y \in\left\{w, b_{2}-\right.$ $\left.s, b_{3}-s\right\}$, depending on the employment state. We define the average discounted probabilities (ADP's) of being in state 2 and 3, respectively, by

$$
\begin{aligned}
& \Pi_{2} \equiv r \int_{0}^{\infty} \mathrm{e}^{-r t} \mu_{2, t} \mathrm{~d} t, \\
& \Pi_{3} \equiv r \int_{0}^{\infty} \mathrm{e}^{-r t} \mu_{3, t} \mathrm{~d} t,
\end{aligned}
$$

where $\mu_{2, t}$ and $\mu_{3, t}$ are the probabilities of being short-term and long-term unemployed at time $t$, respectively, conditional on being employed at time zero, provided that individuals who can move do so and that unemployed search for a job. ${ }^{10}$ The actuarial fairness requirement of the UI system is now a simple linear function of the benefits

$$
\tau=\Pi_{2} b_{2}+\Pi_{3} b_{3} .
$$

\subsection{Value functions and consumption}

Under constant absolute risk aversion and stationary income uncertainty, the value functions for the three states $j \in\{1,2,3\}$ can be separated

$$
V\left(A_{t}, j\right)=W\left(A_{t}\right) \tilde{V}_{j}\left(\tau, b_{2}, b_{3}\right),
$$

where

$$
\begin{aligned}
& W\left(A_{t}\right) \equiv \frac{\mathrm{e}^{-\gamma A_{t}}}{r}, \\
& \tilde{V}_{j} \equiv-\mathrm{e}^{-\gamma c_{j}},
\end{aligned}
$$

and $\sigma_{j}$ are state-dependent consumption constants such that the state dependent consumption functions are

$$
c_{j}\left(A_{t}\right)=r A_{t}+\sigma_{j} .
$$

The consumption constants $\sigma_{j}$ are nonlinear functions of income in all states and thus, depend on the planner choice variables $\tau, b_{2}$ and $b_{3}$. The constants are found as the unique

\footnotetext{
${ }^{9}$ Directly related to the introductory discussion about wealth effects, we note that the CARA specification implies that individual assets do not affect preference over insurance. Older employed agents with non-zero asset holdings would therefore not want to renegotiate their contract.

${ }^{10}$ It is straightforward to calculate that

$$
\begin{aligned}
\Pi_{2} & \equiv \frac{q(1-p)(h+r)}{(r+h+q(1-p))(r+h+f)}, \\
\Pi_{3} & \equiv \Pi_{2} \frac{f}{h+r} .
\end{aligned}
$$
}


solutions to the Bellman equations for each state: ${ }^{11}$

$$
\begin{aligned}
& \sigma_{1}=w-\tau-\frac{q\left(p \mathrm{e}^{\gamma r m}+(1-p) \mathrm{e}^{\gamma \Delta_{2}}-1\right)}{\gamma r}, \\
& \sigma_{2}=b_{2}-s-\tau+\frac{h\left(1-\mathrm{e}^{-\gamma \Delta_{2}}\right)}{\gamma r}-\frac{f\left(\mathrm{e}^{\gamma\left(\Delta_{3}-\Delta_{2}\right)}-1\right)}{\gamma r}, \\
& \sigma_{3}=b_{3}-s-\tau+\frac{h\left(1-\mathrm{e}^{-\gamma \Delta_{3}}\right)}{\gamma r},
\end{aligned}
$$

where

$$
\begin{aligned}
& \Delta_{2} \equiv \sigma_{1}-\sigma_{2}, \\
& \Delta_{3} \equiv \sigma_{1}-\sigma_{3},
\end{aligned}
$$

are the consumption differences between states 1 and 2 and between states 1 and 3 , respectively.

\section{Optimal insurance}

Given the discussion above, the problem we set out to solve is to maximize the ex ante value of unemployment insurance, that is, we want to maximize the welfare of an individual upon entering the economy. This welfare is given by $V(0,1)$, since we assume that agents enter the economy as employed with no assets. ${ }^{12}$ Due to the separability and the fact that $W\left(A_{t}\right)$ is independent of the insurance system, we immediately see that this is equivalent to maximizing $\tilde{V}_{1}$ over $\left\{\tau, b_{2}, b_{3}\right\}$. Using the budget constraint $\tau=\Pi_{2} b_{2}+\Pi_{3} b_{3}$, our objective is therefore to maximize

$$
\tilde{V}_{1}\left(\Pi_{2} b_{2}+\Pi_{3} b_{3}, b_{2}, b_{3}\right)
$$

over $b_{2}$ and $b_{3}$ subject to the incentive constraints that unemployed individuals voluntarily search for a job and that individuals with the opportunity to move to get a job voluntarily do so.

In the direct formulation of the problem, the incentive constraints are highly non-linear functions of the choice variables $b_{2}$ and $b_{3}$. This makes it hard to find the binding constraints, which is necessary to find the solution. However, it turns out that we can formulate the problem so that the incentive constraints are linear and either parallel or orthogonal. Finding out which is binding is then trivial. Furthermore, adding more states and incentive constraints is also very simple. We regard this as the methodological contribution of the paper.

Finding the constrained optimal insurance now involves the following steps:

1. Note that $\tilde{V}_{1} \equiv-\mathrm{e}^{-\gamma c_{1}}$ is a monotone transformation of $\sigma_{1}$. For convenience, we therefore use $\sigma_{1}$ from (6) as the objective function noting that it is a function of the consumption differences. Then use (6) and the budget constraint (2) to express $\tau$ in terms of the consumption differences and finally use this to substitute for $\tau$ in the objective function $\left(\sigma_{1}\right)$.

\footnotetext{
${ }^{11}$ See the appendix for proof that the proposed value and consumption functions solve the Bellman equations.

${ }^{12}$ Obviously, we could equally well have chosen any other initial condition. Note also that the separability implies that the insurance system that maximizes the ex ante utility also maximizes the utility of all employed, regardless of their history.
} 
2. Express the incentive constraints in terms of consumption differences $\Delta_{j}$.

3. Maximize $\sigma_{1}$ over the consumption differences, subject to the incentive constraints.

4. Verify that the optimal consumption differences $\Delta_{2}$ can be implemented by some combination of $b_{j}$ 's.

\subsection{Two states}

For illustrative purposes, we start with the simplest case of two states, i.e., we assume that $f=0$ so unemployment benefits are constant forever.

The first step is now to derive an expression for $\sigma_{1}$ in terms of $\Delta_{2}$ where the budget constraint (2) is used to replace the tax rate. For this purpose, we subtract the second line of (6) from the first and solve for $b_{2}$. Then, we use this expression in the budget constraint $\tau=\Pi_{2} b_{2}$ and substitute for $\tau$ in the first line of (6). This yields

$$
\sigma_{1}=\kappa+\Pi_{2}\left(\Delta_{2}-\frac{h \mathrm{e}^{-\gamma \Delta_{2}}}{\gamma r}\right)-\left(1-\Pi_{2}\right) q \frac{(1-p) \mathrm{e}^{\gamma \Delta_{2}}}{\gamma r},
$$

where $\kappa$ is a constant, independent of the choice variables. Straightforward calculus shows that (9) defines $\sigma_{1}$ as a concave function of $\Delta_{2}$ with a unique maximum at 0 . The reason for $\sigma_{1}$ being maximized at $\Delta_{2}=0$ is obvious - when actuarial insurance is available, full insurance maximizes utility. However, $\Delta_{2}=0$ is not incentive compatible. Neither searching nor moving will occur voluntarily under full insurance. Therefore, we turn to step 2-where we find the incentive constraints.

The ICM constraint implies that a person who has lost her job and has the opportunity to move must be induced to do so. We first note that if her assets upon separation were $A_{t}$, her value immediately after moving is

$$
V\left(A_{t}-m, 1\right)=-\frac{1}{r} \mathrm{e}^{-\gamma r\left(A_{t}-m\right)} \mathrm{e}^{-\gamma \sigma_{1}},
$$

since she has paid the moving cost, $m$. We compare this to the value of a one-period deviation, i.e., the value if the individual does not move during this unemployment spell. Immediately after being laid off, her assets are $A_{t}$ and she is unemployed, i.e., in state 2, since she did not take the opportunity to move to get a job. Her value is therefore,

$$
V\left(A_{t}, 2\right)=-\frac{1}{r} \mathrm{e}^{-\gamma r A_{t}} \mathrm{e}^{-\gamma \sigma_{2}} .
$$

To induce moving, we need $V\left(A_{t}-m, 1\right) \geqslant V\left(A_{t}, 2\right)$. It immediately follows that this requires

$$
\Delta_{2} \geqslant r m
$$

We label (10) the ICM-condition.

Now, consider the incentive to search. Remember that for now, we assume unemployment benefits to be flat (the assumption $f=0$ implies that $b_{3}$ is irrelevant). If the individual does not search, she therefore gets an income $b_{2}-\tau$ for ever, since she will not find a new job without searching. Without uncertainty, she consumes exactly her total income $r A_{t}+b_{2}-\tau$ (since $r$ coincides with the subjective discount rate) and her utility is therefore

$$
-\frac{1}{r} \mathrm{e}^{-\gamma r A_{t}} \mathrm{e}^{-\gamma\left(b_{2}-\tau\right)} .
$$




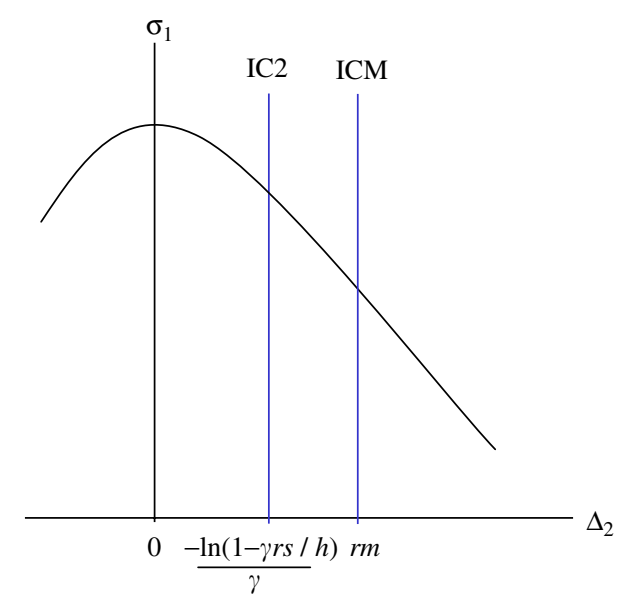

Fig. 1. Objective function and constraints in a two-state case.

The utility if the individual instead searches is $-(1 / r) \mathrm{e}^{-\gamma r A_{t}} \mathrm{e}^{-\gamma \sigma_{2}}$ so to induce search, we clearly need

$$
\sigma_{2} \geqslant b_{2}-\tau
$$

Note that the consumption of the unemployed who search is $r A_{t}+\sigma_{2}$. Furthermore, her total income net of search costs is $r A_{t}+b_{2}-\tau-s$. Therefore, the search condition implies consumption to be strictly higher than income. Over time, the unemployed depletes her assets and consumption therefore falls, despite the benefits being constant. The celebrated result by Shavell and Weiss (1979) and Hopenhayn and Nicolini (1997) that consumption should optimally fall over the unemployment spell when the insurer can fully control consumption (no hidden savings) is therefore mimicked in this case, where hidden savings are allowed.

The final part of step 2 is to express the search constraint in terms of the consumption difference $\Delta_{2}$. Using the second line of (6) and setting $f=0$, the search constraint can be written

$$
\Delta_{2} \geqslant-\frac{\ln (1-\gamma r s / h)}{\gamma},
$$

which we label the IC2-condition. As can be seen, the incentive constraints are simply constants and it is immediate to see which one is binding.

The problem is now simply depicted in Fig. 1, where we note that the two constraints are parallel.

In the depicted case, it is the ICM-constraint that binds and step 3 is trivial. Maximizing $\sigma_{1}$ over $\Delta_{2}$ subject to the ICM constraint implies

$$
\Delta_{2}=r m \text {. }
$$

Finally, we want to implement this. This is easily done using (6); set the difference between the first and the second line equal to $\mathrm{rm}$ and solve for $b_{2}$, giving

$$
b_{2}=w+s-r m-\frac{q\left(\mathrm{e}^{\mathrm{\gamma} r m}-1\right)+h\left(1-\mathrm{e}^{-\gamma r m}\right)}{\gamma r} .
$$


In the alternative case, where the IC2 constraint binds, we instead get

$$
b_{2}=w+\frac{\ln \left(1-\gamma r \frac{s}{h}\right)}{\gamma}-\frac{s q}{h-\gamma r s},
$$

where both expressions are unique and easily lend themselves to comparative statics.

\subsection{Three states}

The procedure in the case of three states is exactly analogous to the two-state case and simply extends to any number of finite states. We use (6) and the budget constraint (2) to express $\sigma_{1}$ as a function of the consumption differences, now $\Delta_{2}$ and $\Delta_{3} \equiv \sigma_{1}-\sigma_{3}$ (step 1). Then, we express the incentive constraints in terms of $\Delta_{2}$ and $\Delta_{3}$, check which are binding (step 2), maximize $\sigma_{1}$ over $\left\{\Delta_{2}, \Delta_{3}\right\}$ subject to the binding constraints (step 3 ) and find the implementing $b_{2}, b_{3}$ (step 4 ).

\subsubsection{Objective and constraints}

Using the equations for the consumption constants (6) and the budget constraint (2), the objective becomes

$$
\begin{aligned}
\sigma_{1}= & \kappa_{2}+\Pi_{2} \Delta_{2}+\Pi_{3} \Delta_{3}-\left(1-\Pi_{2}-\Pi_{3}\right) \frac{q(1-p)}{\gamma r} \mathrm{e}^{\gamma \Delta_{2}} \\
& -\Pi_{2}\left(h \frac{\mathrm{e}^{-\gamma \Delta_{2}}}{\gamma r}+f \frac{\mathrm{e}^{\gamma\left(\Delta_{3}-\Delta_{2}\right)}}{\gamma r}\right)-\Pi_{3} h \frac{\mathrm{e}^{-\gamma \Delta_{3}}}{\gamma r},
\end{aligned}
$$

where $\kappa_{2}$ is an unimportant constant. In Fig. 2, we make a graphical representation of the objective function by drawing indifference curves in a figure with $\Delta_{3}$ on the $x$-axis and $\Delta_{2}$ on the $y$-axis. ${ }^{13}$ The bliss point is at full insurance, when $\left\{\Delta_{3}, \Delta_{2}\right\}=\{0,0\}$, again, for the reason that the insurance is actuarially fair. The indifference curves have elliptical shapes around the bliss point, of which we are only interested in the segment in the positive quadrant, since incentive compatibility certainly requires $\Delta_{3}, \Delta_{2} \geqslant 0$. For the later analysis, we should note that the slope of an indifference curve is strictly positive if $\Delta_{3}=0$ and $\Delta_{2}>0$ and that it is downward sloping at $\Delta_{2}=\Delta_{3}$, regardless of the parameter choice. ${ }^{14}$

Regarding the three incentive constraints, it is straightforward to see that they are identical to the case of two states, ${ }^{15}$ i.e., the ICM is $\Delta_{2} \geqslant r m$ and the IC2 and IC 3 constraints are

$$
\Delta_{2}, \Delta_{3} \geqslant-\gamma^{-1} \ln \left(1-\gamma r \frac{s}{h}\right) \text {. }
$$

The intuition for the fact that IC2 and IC3 are identical is simple. In our base line case, hiring probabilities and search costs of searching individuals are the same for long- and shortterm unemployed. The incentives in terms of utility and thus, in terms of consumption increases upon successful search, must therefore be the same. Allowing different search costs and/or hiring probabilities in the two states is, however, very simple by allowing $s$ and $h$ to

\footnotetext{
${ }^{13}$ The indifference curves in Figs. 2-6 are drawn for $\{h=1, f=1, q=0.1, r=0.05, \gamma=1, p=0.5\}$ but the results below hold for all parameter values.

${ }^{14}$ Differentiating the objective function, we find the derivative of the indifference curve to be $\frac{f \mathrm{e}^{-\gamma \Delta_{2}}}{r+(h+f)\left(1+\mathrm{e}^{\left.-\gamma \Delta_{2}\right)}\right.} \in$ $(0,1)$ at $\Delta_{3}=0$ and $\frac{-\mathrm{e}^{-\gamma \Delta_{2}}}{1+\frac{r}{h}+\frac{(h+r)^{2}}{f h}+\frac{(h+r)\left(\mathrm{e}^{\left.-\gamma \Delta_{2}\right)}\right.}{f}} \in(-1,0)$ at $\Delta_{2}=\Delta_{3}$.

${ }^{15}$ See the appendix for a formal proof.
} 


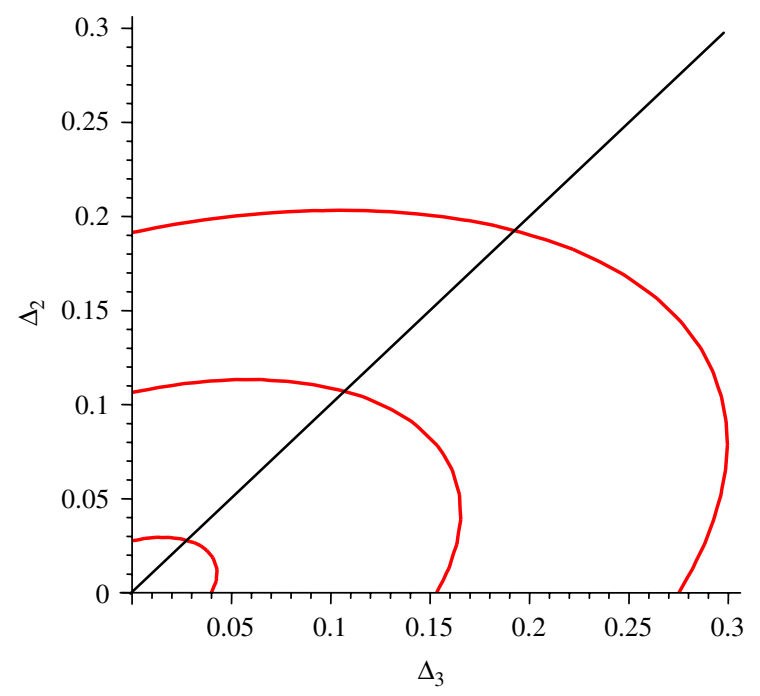

Fig. 2. Indifference curves.

be state dependent in the IC conditions; this is done in Section 5.3. Therefore, we reach the key conclusion that the incentive constraints for the two states (IC2 and IC3) are identical and orthogonal in the $\left\{\Delta_{2}, \Delta_{3}\right\}$-space. We emphasize that this does not mean that only $b_{2}\left(b_{3}\right)$ is of importance for search incentives of the short-term (long-term) unemployed. On the contrary, both $b_{2}$ and $b_{3}$ affect consumption and therefore incentives in all states. However, individual optimization and access to markets for saving and borrowing imply that the value function is a monotonous transformation of consumption. Thus, the wedge between consumption in the current state and during employment is a sufficient statistic to determine whether search incentives are sufficiently strong.

In the next subsection, we will use our model to characterize the optimal UI-scheme under different assumptions on which the constraint is binding. As in the two-state case, the analysis is greatly simplified by the incentive constraints in $\left\{\Delta_{3}, \Delta_{2}\right\}$ space being linear and parallel or orthogonal. When the optimal $\left\{\Delta_{2}, \Delta_{3}\right\}$ are found, we find the optimal benefits from the implementation mapping, which is derived by taking the difference between lines 1 and 2 and between 1 and 3 in (6) and solving for $b_{2}$ and $b_{3}$ :

$$
\begin{aligned}
b_{2}= & w+s-\Delta_{2} \\
& -\frac{q\left(p \mathrm{e}^{\gamma r m}+(1-p) \mathrm{e}^{\gamma \Delta_{2}}-1\right)+h\left(1-\mathrm{e}^{-\gamma \Delta_{2}}\right)-f\left(\mathrm{e}^{\gamma\left(\Delta_{3}-\Delta_{2}\right)}-1\right)}{\gamma r}, \\
b_{3}= & w+s-\Delta_{3}-\frac{q\left(p \mathrm{e}^{\gamma r m}+(1-p) \mathrm{e}^{\gamma \Delta_{2}}-1\right)+h\left(1-\mathrm{e}^{-\gamma \Delta_{3}}\right)}{\gamma r} .
\end{aligned}
$$

\section{Characterization of optimal UI-schemes}

In this section, we use our model to characterize (constrained) optimal unemployment insurance if search cost are low and high, respectively. In the following section, we will extend the analysis in a few directions. 


\subsection{Small search costs}

We start the analysis with the assumption that search costs are sufficiently small to be ignored, later they are re-introduced. First, we analyze the problem graphically by including the ICM constraint, i.e., $\Delta_{2} \geqslant r m$ in the indifference curve graph (Fig. 3), and then we provide analytical results.

The ICM constraint is satisfied for all values of $\Delta_{2}$ above horizontal constraint. The optimizing choice of $\Delta_{3}$ is where the ICM constraint is tangent to an indifference curve. This occurs for the solid indifference curve in Fig. 3. As noted above, the indifference curve is positively sloped at $\Delta_{3}=0$ and negatively sloped at $\Delta_{2}=\Delta_{3}$ implying that the tangency must be at a point where $\Delta_{3}>0$ and $\Delta_{3}<\Delta_{2}$. This means that state 2 should be "worse" than state 3 in the sense that, given assets, utility and consumption are higher in state 3 than in state 2. It is intuitive (and easily proved) that $\Delta_{2}>\Delta_{3}>0$ implies that $b_{2}-$ $s<b_{3}-s<w$. The intuition for this is that when $b_{2}-s=b_{3}-s$, the two unemployment states are, by construction, identical so that $\Delta_{2}=\Delta_{3}$. Making $\Delta_{2}$ larger than $\Delta_{3}$ requires a reduction in benefits for short-term unemployed and/or an increase in benefits for longterm unemployed.

Result 1. If search costs are sufficiently low, only the ICM constraint is binding and benefits should optimally increase over time.

The economic reason for our results can be phrased in the following way. To separate individuals who have the option of moving from those who have not such an option, a positive $\Delta_{2}$ is required. However, this does not call for an inefficient structure of the benefit schedule. Specifically, starting from a flat benefit schedule (along the $45^{\circ}$ line where $\Delta_{2}=\Delta_{3}$ ), the welfare in all states can be increased, while maintaining the necessary wedge $\Delta_{2}=r m$, by increasing benefits for long-term unemployed and reducing benefits for shortterm unemployed. The reason for this is that the expected marginal utility is higher for individuals who have been unemployed for a long time. The optimum is, however, reached before benefits to long-term unemployed are sufficiently high to make the latter indifferent

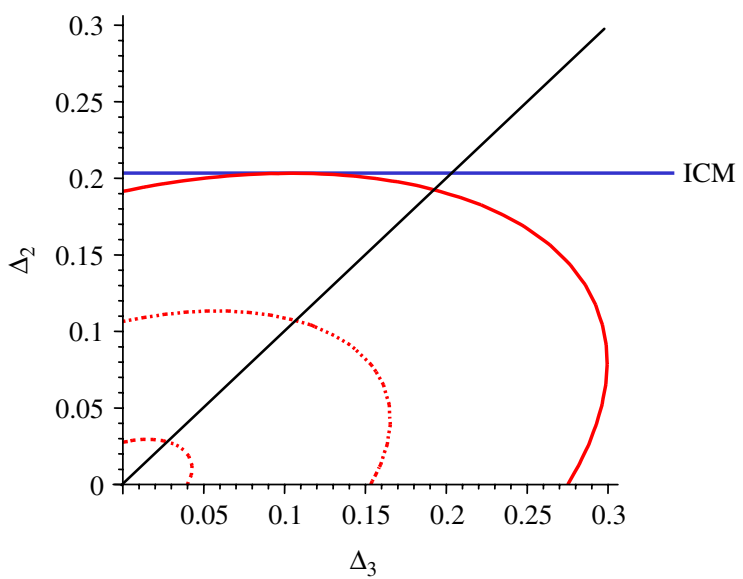

Fig. 3. Indifference curves and Incentive Constraint for Moving (ICM). 
between having a job and remaining unemployed. On the other hand, when $\Delta_{3}=0$ while $\Delta_{2}=r m$, long-term unemployed are as well off as the employed (given assets) and their expected marginal utility is relatively low. A reallocation from long-term to short-term benefits therefore increases the value of the insurance so that the tax-cost of providing a given insurance value can be reduced.

Now, let us derive closed-form solutions to our problem. Using the binding ICM condition $\Delta_{2}=\mathrm{rm}$ to substitute for $\Delta_{2}$, the objective function (14) simplifies and the problem can then be written

$$
\max _{\Delta_{3} \in R^{+}}\left\{\Pi_{3}\left(\Delta_{3}-h \frac{\mathrm{e}^{-\gamma \Delta_{3}}}{\gamma r}\right)-\Pi_{2} f \frac{\mathrm{e}^{\gamma\left(\Delta_{3}-r m\right)}}{\gamma r}\right\} .
$$

These terms have straightforward interpretations. The first term is due to the benefit of reducing the tax-cost of long-term benefits. This term is increasing in $\Delta_{3}$, since higher $\Delta_{3}$ is achieved by lower benefits for long-term unemployed, which reduce taxes in proportion to the ADP of long-term unemployment, $\Pi_{3}$. Note that this tax reduction comes from two sources; there is a direct effect that is proportional to $\Delta_{3}$ but there is also an indirect effect, captured by the second term inside the parenthesis. Long-term unemployed find jobs at a positive rate, $h$. The prospect of finding a job keeps up consumption, so that it falls less than proportionally to the reduction in benefits. Conversely, given an increase in $\Delta_{3}$, benefits can be reduced more than proportionally.

The second term in (17) is due to the benefit of reducing the tax cost of short-term benefits. It is decreasing in $\Delta_{3}$ since less consumption for long-term unemployed has a negative impact on consumption also of the short-term unemployed, proportional to $f$. As $\Delta_{3}$ increases, benefits to the short-term unemployed must therefore increase to keep $\Delta_{2}=$ $\mathrm{rm}$. This has a tax-cost proportional to the ADP of short-run unemployment $\Pi_{2}$.

The objective function in (17) is concave in $\Delta_{3}$. Thus, the unique solution to the problem is obtained by the solution to the first-order condition, given by

$$
\Delta_{3}^{*}=-\frac{\ln \left(\sqrt{\left(\frac{r}{2 h}\right)^{2}+\mathrm{e}^{-\gamma r m}\left(\frac{h+r}{h}\right)}-\frac{r}{2 h}\right)}{\gamma}>0 .
$$

Using the implementation mapping (16), we can find the optimal insurance scheme. In particular, in optimum

$$
b_{3}^{*}-b_{2}^{*}=r m-\Delta_{3}^{*}+\left(f+h \mathrm{e}^{-\gamma \Delta_{3}^{*}}\right) \frac{1-\mathrm{e}^{-\gamma\left(r m-\Delta_{3}^{*}\right)}}{\gamma r}>0 .
$$

Notice also that since the solution for $\Delta_{3}$ is independent of $f$, the difference $b_{3}-b_{2}$ should increase in $f$. It can be shown that the derivative of the objective function with respect to $f$ is always positive. Low values of $f$ is an inefficient way of inducing separation between those who can move and those who cannot, as agents expect to spend a longer stochastic time suffering the low short-run benefits. Without formally showing this, we conjecture that if lump-sum benefits were allowed, the best policy would be to punish unemployment by a lump-sum unemployment tax when an individual becomes unemployed. In reality, however, it may be politically difficult or even infeasible to implement a lump-sum punishment on those who lose their jobs. Furthermore, a lower 
bound on $b_{2}$, for example zero, might be imposed for political reasons, in which case this would pin down an optimal $f$ from (18).

As is clear from the above analysis, a reduction in $m$ reduces $\Delta_{2}$ and allows a more generous unemployment insurance. Such a reduction could be achieved by subsidies to moving or retraining. However, full compensation is unlikely to be optimal in reality. Suppose, realistically, that individuals with a job sometimes experience a preference or productivity shock, making another job or a job in another location more attractive than the current one. Suppose also that these shocks are not sufficiently large to induce voluntary separation and moving if the individual must pay the moving cost herself. Clearly, such moves are then not socially optimal. The insurer would like to fully subsidize the moving cost of individuals who are involuntarily separated from their job, but not subsidize it for individuals who voluntary separate to claim the subsidy. However, this is infeasible if the insurer cannot distinguish voluntary and involuntary separations. Therefore, we argue that although partial subsidies may be feasible and, in fact, observed in reality, full subsidization is unrealistic. More specifically, it seems clear that subsidies should be as large as possible, without inducing inefficient voluntary separation. Thus, we could interpret $m$ as the cost of moving or retraining, net the optimal subsidy. Furthermore, a large subsidy to moving might lead unemployed individuals to claim the subsidy, which is likely to be inefficient. This issue is analyzed below in Section 5.4.

\subsection{Larger search costs}

We can now easily analyze the conditions such that IC2 and IC3 are satisfied, despite positive search costs. Graphically, the constraints are simply horizontal and vertical lines and all values of $\Delta_{2}\left(\Delta_{3}\right)$ above (to the right of) these lines imply that the respective constraints are satisfied. If search costs are sufficiently small, none of the search constraints bind, as shown in Fig. 4, where IC2 is slack while IC3 almost binds at the tangency between ICM and an indifference curve. This occurs at the point indicated by the arrow on the solid indifference curve.

Increasing search costs shift out IC2 and IC3 since from (15) we see that the RHS is increasing in $s$. Eventually (for a search cost which is sufficiently large) IC3 is no longer satisfied at the point where the ICM constraint is tangent to the indifference curve. This situation is depicted in Fig. 5. Here, the point where the ICM is tangent to the most outward dotted indifference curve satisfies the IC2 constraint, but not the IC3 constraint. Thus, $\Delta_{3}$ must be increased but since the IC3 and the ICM constraint are orthogonal, $\Delta_{2}$ need not be changed. The optimal point is where the ICM and the IC3 constraint cross. This point is indicated by the arrow and on the solid indifference curve. Clearly, $\Delta_{3}$ remains smaller than $\Delta_{2}$ implying an upward sloping benefit profile, i.e., $b_{2}<b_{3}$. Specifically, $\Delta_{2}$ should be set equal to $r m$ and $\Delta_{3}$ equal to $-\gamma^{-1} \ln (1-\gamma r s / h)$. This means that individuals will be indifferent in the choice of moving and that long-term unemployed are indifferent to searching, while the short-term unemployed strictly prefer to search.

Result 2. For an intermediate range of search costs, the ICM and the IC3 constraints are binding and benefits should optimally increase over time.

A further increase in search costs will eventually call for a situation like that in Fig. 6. Here, both search constraints bind, while the moving constraint is slack. Once more, the 


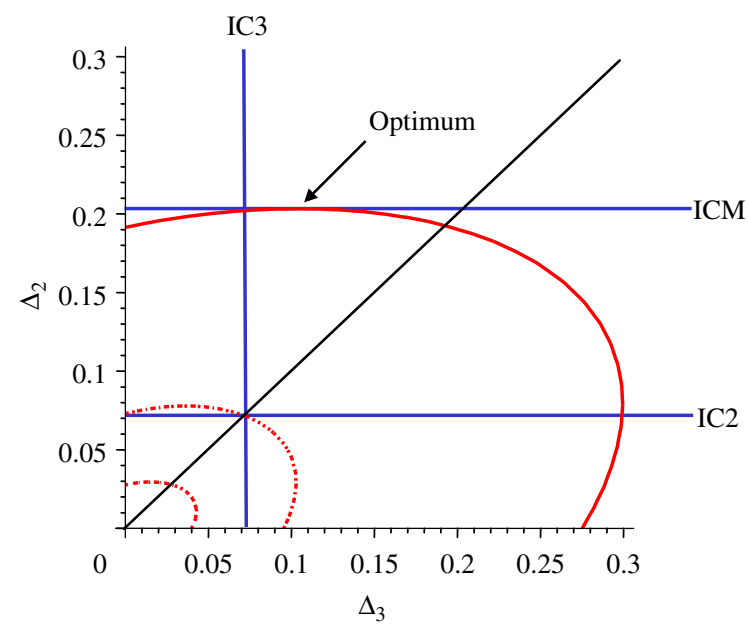

Fig. 4. Low search costs.

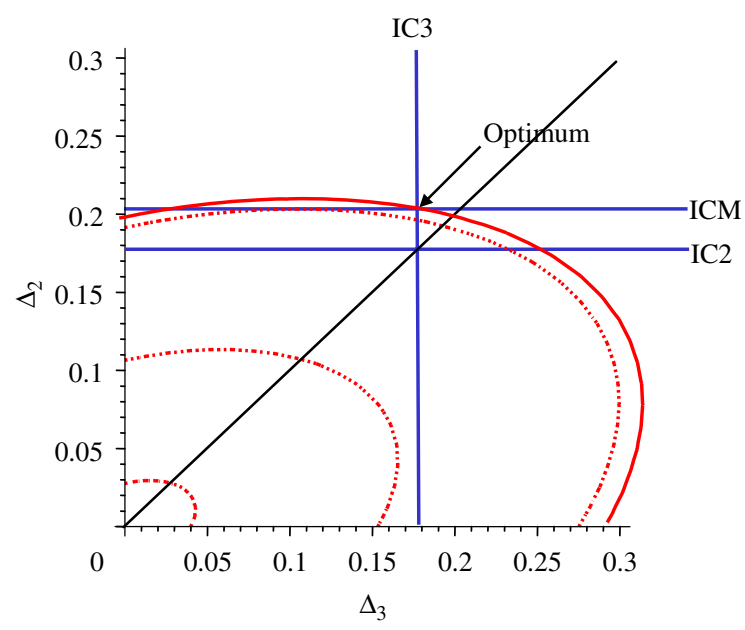

Fig. 5. Moderate search costs.

optimum is indicated by the arrow and on the solid indifference curve. Benefits are constant and given by expression (13) since $\Delta_{2}=\Delta_{3}=-\gamma^{-1} \ln (1-\gamma r s / h) .{ }^{16}$ We conclude:

Result 3. For sufficiently high search costs, the IC2 and the IC3 constraints are binding and benefits should optimally be constant over time.

The conclusion so far is that when the moving cost is large relative to the search costs, then the optimal unemployment insurance scheme involves an increasing benefit profile in order to, on the one hand, generate incentives to move for those agents who can and, on

\footnotetext{
${ }^{16}$ This is a special case of results in Werning (2002) and Shimer and Werning (2005) showing that constant benefits are optimal under CARA utility in a general class of UI-schemes.
} 


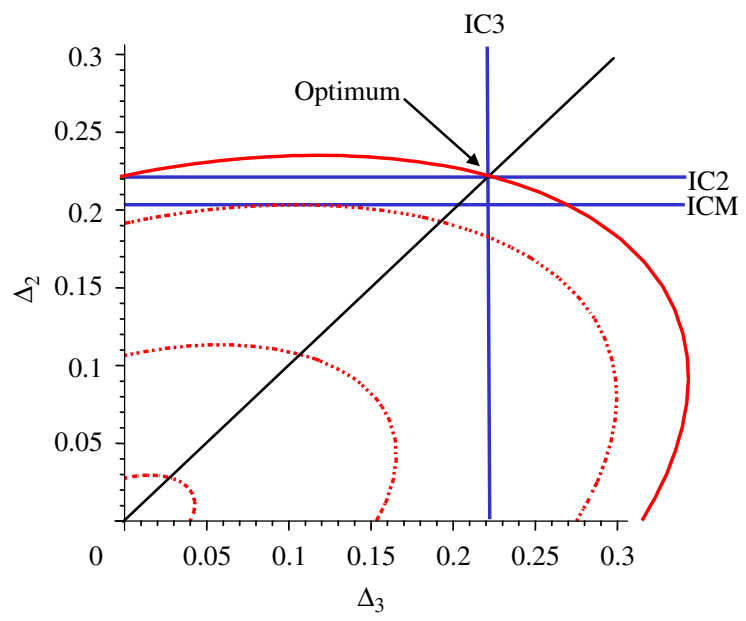

Fig. 6. High search costs.

the other hand, not too much limiting insurance for the possibility that an unemployment period becomes long-lasting.

If the search costs are sufficiently high relative to the moving cost, strong search incentives are needed and the moving constraint is slack. In this case, the optimal benefit profile is flat. The intuition behind this result is that, on the one hand, search incentives are strengthened by falling benefits. On the other hand, when private savings are allowed, buffer stock savings provide a good substitute for short but not for long unemployment spells, calling for an upward sloping benefit profile. These two effects cancel exactly under CARA utility. With other utility functions both effects are present but will in general not cancel each other. ${ }^{17}$

\section{Extensions}

In this section, we will extend and generalize the model in a few directions. We show that the applicability of the approach is more general than to the particular case analyzed above.

\subsection{Continuously arriving moving opportunities}

The analysis in the previous section was done under the assumption that moving opportunities arise immediately upon separation. In this subsection, we relax this assumption, now allowing a finite arrival rate of moving opportunities. As above, we allow heterogeneity among unemployed in the sense that only a share $p$ of individuals who loose their job might eventually receive such moving opportunities. We call such individuals unemployed of type $M$ (movers) while individuals who will never get moving opportunities are called unemployed of type $S$ (stayers).

\footnotetext{
${ }^{17}$ See Hassler and Rodríguez Mora (1999) for an analysis of the relative value of insurance against long and short unemployment spells under CARA and CRRA utility.
} 
Unemployed of type $M$ get opportunities to move with an arrival rate $\lambda$, provided that they exert search effort. If such an opportunity arises, the individual choose if she wants to pay the moving cost $m$, in which case she immediately gets a job. All unemployed individuals, regardless of type, also get job opportunities with arrival rate $h$, provided that they exert search effort. The labor market status can now take 5 values, employed (1), short-term unemployed mover (M, 2), long-term unemployed mover (M, 3), short-term unemployed stayer $(\mathrm{S}, 2)$ and long-term unemployed stayer $(\mathrm{S}, 3)$. The corresponding consumption constants are denoted $\left\{\sigma_{1}, \sigma_{M, 2}, \sigma_{M, 3}, \sigma_{S, 2}, \sigma_{S, 3}\right\}$ and in direct analogy with (6) they must satisfy

$$
\begin{aligned}
& \sigma_{1}=w-\tau-q \frac{\left(p \mathrm{e}^{\gamma \Delta_{M, 2}}+(1-p) \mathrm{e}^{\gamma \Delta_{S, 2}}-1\right)}{\gamma r}, \\
& \sigma_{S, 2}=b_{2}-s-\tau+\frac{h\left(1-\mathrm{e}^{-\gamma \Delta_{S, 2}}\right)}{\gamma r}-\frac{f\left(\mathrm{e}^{\gamma\left(\Delta_{S, 3}-\Delta_{S, 2}\right)}-1\right)}{\gamma r}, \\
& \sigma_{S, 3}=b_{3}-s-\tau+\frac{h\left(1-\mathrm{e}^{-\gamma \Delta_{S, 3}}\right)}{\gamma r}, \\
& \sigma_{M, 2}=b_{2}-s-\tau+\lambda \frac{\left(1-\mathrm{e}^{-\gamma\left(\Delta_{M, 2}-r m\right)}\right)}{\gamma r}+\frac{h\left(1-\mathrm{e}^{-\gamma \Delta_{M, 2}}\right)}{\gamma r}-\frac{f\left(\mathrm{e}^{\gamma\left(\Delta_{M, 3}-\Delta_{M, 2}\right)}-1\right)}{\gamma r}, \\
& \sigma_{M, 3}=b_{3}-s-\tau+\lambda \frac{\left(1-\mathrm{e}^{-\gamma\left(\Delta_{M, 3}-r m\right)}\right)}{\gamma r}+\frac{h\left(1-\mathrm{e}^{-\gamma \Delta_{M, 3}}\right)}{\gamma r},
\end{aligned}
$$

where $\Delta_{j, k} \equiv \sigma_{1}-\sigma_{j, k}$ for $j \in\{S, M\}$ and $k \in\{1,2\}$.

Now, it is immediate that the incentive constraints for moving is $\Delta_{M, 2}, \Delta_{M, 3} \geqslant r m$ i.e., that utility increases if an option to move is executed at cost $m$. Furthermore, if $r m \geqslant-\ln \left(1-\gamma r \frac{s}{h}\right) / \gamma$, the moving constraints will bind, i.e., $\Delta_{M, 2}=\Delta_{M, 3}=r m$. Since moving provides no extra utility, the utility of stayers is the same as of movers, as is the value of searching. Given that the moving constraints bind, all incentive constraints for search are identical given by

$$
\Delta_{j, k} \geqslant-\frac{\ln \left(1-\gamma r \frac{s}{h}\right)}{\gamma},
$$

which are satisfied under the assumption $r m \geqslant-\ln \left(1-\gamma r \frac{s}{h}\right) / \gamma$.

In Fig. 7 we illustrate this case. We label the ICM constraints for short-term and long term unemployed by ICM2 and ICM3, respectively. These constraints are satisfied in the area above and to the right of ICM2 and ICM3. The fact that the indifference curves are negatively sloped along the $45^{\circ}$ line implies that welfare is maximized at the corner $\Delta_{j, k}=r m$, indicated by the arrow. In this case, benefits are constant at the level given by $(12){ }^{18}$

${ }^{18}$ To see this, substitute $\mathrm{rm}$ for all $\Delta_{j, k}$ in (19) which then reduces to

$$
\begin{aligned}
& \sigma_{1}=w-\tau-q \frac{\left(\mathrm{e}^{\gamma r m}-1\right)}{\gamma r}, \\
& \sigma_{j, k}=b_{2}-s-\tau+\frac{h\left(1-\mathrm{e}^{-\gamma r m}\right)}{\gamma r} .
\end{aligned}
$$

Set the difference to $\mathrm{rm}$ and solve for $b_{2}$. 


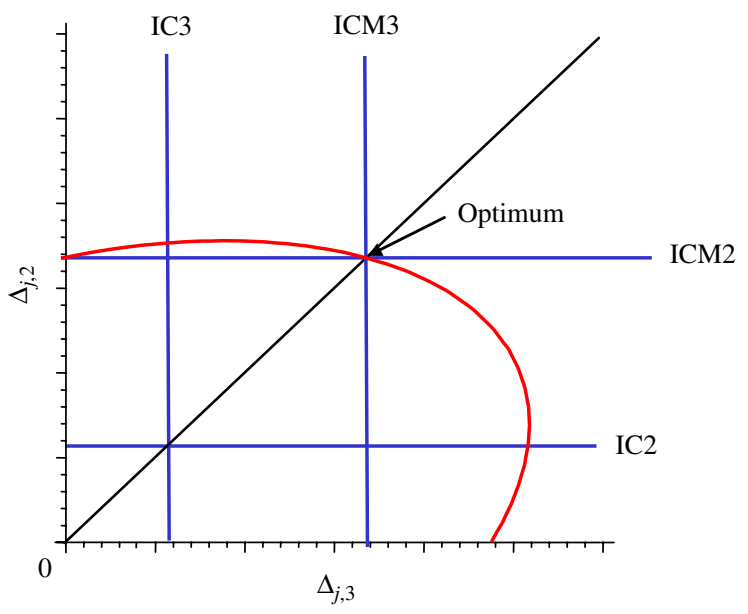

Fig. 7. The moving constraints bind for both short-term and long-term unemployed.

If search cost are sufficiently high, i.e., $r m<-\ln \left(1-\gamma r \frac{s}{h}\right) / \gamma$, the moving constraints (ICM2 and ICM3) are slack while the search constraints (IC2 and IC3) of the stayers will bind. The search constraints of the movers will be slack. The reason for this is that the value of search is larger for movers due to the fact that an extra benefit of searching for them is that it generates a flow of moving opportunities with positive value. In this case, the solution is at the corner given by $\Delta_{S, 2}=\Delta_{S, 3}=-\ln \left(1-\gamma r \frac{s}{h}\right) / \gamma$. There is then no closed form solution for $b_{2}$, but it can easily be found numerically by substituting $\Delta_{S, 2}=\Delta_{S, 3}=$ $-\ln \left(1-\gamma r \frac{s}{h}\right) / \gamma$ into (19) noting that $\Delta_{M, 2}=\Delta_{M, 3}$ and solving for the values of $\Delta_{M, 2}$ and $b_{2}$.

Our conclusion so far in this subsection is that if moving opportunities arrive at a finite rate, this adds an incentive constraint associated with long-term unemployed moving - a second ICM constraint. This new constraint implies that benefits should be constant over time.

The cost of not satisfying the added constraint is that long-term unemployed who get moving opportunities decline these, which ceteris paribus increases taxes. However, if $\lambda$ is high relative to $f$, the mass of long-term unemployed of type $M$ (movers) is small and the increased tax burden associated with not meeting their incentive constraint may be small. We should note that this contrasts sharply with a violation of the search constraint for the long-term unemployed. If this is not satisfied, every individual in the economy will eventually end up unemployed forever.

The potentially lower cost of violating the second ICM constraint implies that we may need to check whether welfare actually is higher if this incentive constraint is dropped. In such a case we are back to the analysis in the previous section. Numerical methods are most convenient to define the set of parameters for which this is the case. ${ }^{19}$ An interesting potential consequence of violating the second ICM constraint is that this provides an argument for reducing $f$, i.e., increasing the duration of short-term benefits. By doing this, a larger share of unemployed of type $M$ will have had time to find a moving opportunity and left unemployment. We conjecture that for this reason, there may in this case be an interior solution to the optimal choice of $f$.

\footnotetext{
${ }^{19}$ It should be noted that if $p$ is low and $m$ large, it could for the same reason be optimal to disregard all incentive constraints for moving.
} 


\subsection{Continuous search effort}

We have so far assumed that search effort is dichotomous, either high or zero. This has made it possible to define incentive compatibility (IC) constraints for search. In reality, however, it may perhaps be more reasonable to assume that search effort is a continuous variable. In this subsection, we will therefore assume that unemployed can choose the hiring rate and that the search cost is an increasing and convex function of the hiring rate, denoted $s(h)$. We can now no longer define IC constraints as in the previous section. Instead, there is a smooth trade-off between insurance and search incentives.

The consumption constants still satisfy (6), with $s$ replaced by $s(h)$. Furthermore, shortterm and long-term unemployed choose their hiring rate, denoted $h_{2}$ and $h_{3}$, respectively. The first-order conditions for these choices are

$$
\begin{aligned}
& s^{\prime}\left(h_{2}\right)=\frac{1-\mathrm{e}^{-\gamma \Delta_{2}}}{\gamma r}, \\
& s^{\prime}\left(h_{3}\right)=\frac{1-\mathrm{e}^{-\gamma \Delta_{3}}}{\gamma r} .
\end{aligned}
$$

The fact that $h_{2}$ and $h_{3}$ may differ affects the calculation of $\Pi_{2}$ and $\Pi_{3}$, now becoming ${ }^{20}$

$$
\begin{aligned}
\Pi_{2} & =\frac{\left(h_{3}+r\right)(1-p) q \rho_{2} \rho_{1}}{\left(r-\rho_{2}\right)\left(r-\rho_{1}\right)\left((1-p) q\left(f+h_{3}\right)+h_{3}\left(f+h_{2}\right)\right)} \\
\Pi_{3} & =\Pi_{2} \frac{f}{h_{3}+r}
\end{aligned}
$$

where $\rho_{1}, \rho_{2}$ are the roots of the dynamic system for $\mu_{2, t}$ and $\mu_{3, t}$. Since the privately chosen $h_{2}$ and $h_{3}$ depend on $\Delta_{2}$ and $\Delta_{3}$, so do $\Pi_{2}$ and $\Pi_{3}$. Increasing $\Delta_{2}$ and $\Delta_{3}$, i.e., reducing the value of unemployment insurance, now has a smooth positive effect on hiring rates and thereby a negative effect on the tax rate. Generically, the optimal unemployment insurance in absence of incentive constraints for moving will therefore, as is well known, involve strictly positive values of $\Delta_{2}$ and $\Delta_{3}$, i.e., less than full insurance. As before, we can construct indifference curves in $\Delta_{2}, \Delta_{3}$ space and in this space introduce the ICM constraint, $\Delta_{2}=r m$. This is done in Fig. $8 .^{21}$

In contrast to the case in the previous section, indifference curves are now centered around a bliss-point with strictly positive $\Delta_{2}$ and $\Delta_{3}$ since the negative effect on search is taken into account. The ICM constraint binds if it is above the bliss point $(r m>0.053$ in the graph). If, in addition, the tangency between the ICM constraint and the relevant indifference curve occurs above the $45^{\circ}$ line, optimal unemployment insurance requires $\Delta_{2}>\Delta_{3}$, i.e., $b_{2}<b_{3}$. We have not been able to prove that this is always going to be the case. ${ }^{22}$ However, we do show that it is a possibility.

\footnotetext{
${ }^{20}$ To derive these, we solve the linear system of differential equations governing the dynamics of $\mu_{2, t}$ and $\mu_{3, t}$ using $\mu_{2,0}=\mu_{3,0}=0$ as initial conditions. After solving this, it is immediate to calculate $r \int_{0}^{\infty} \mathrm{e}^{-r t} \mu_{2, t} \mathrm{~d} t$ and $r \int_{0}^{\infty} \mathrm{e}^{-r t} \mu_{3, t} \mathrm{~d} t$. Proof available upon request.

${ }^{21}$ We used the effort cost function $s(h)=s h^{2} / 2$ and the parameters; $f=1, q=0.1, r=0.05, \gamma=1, p=.5$, $w=1, m=4, s=1$.

${ }^{22}$ The results in Werning (2002) and Shimer and Werning (2005) imply that the bliss-point occurs at the $45^{\circ}$ line. We conjecture that this implies that indifference curves are negatively sloped along this line.
} 


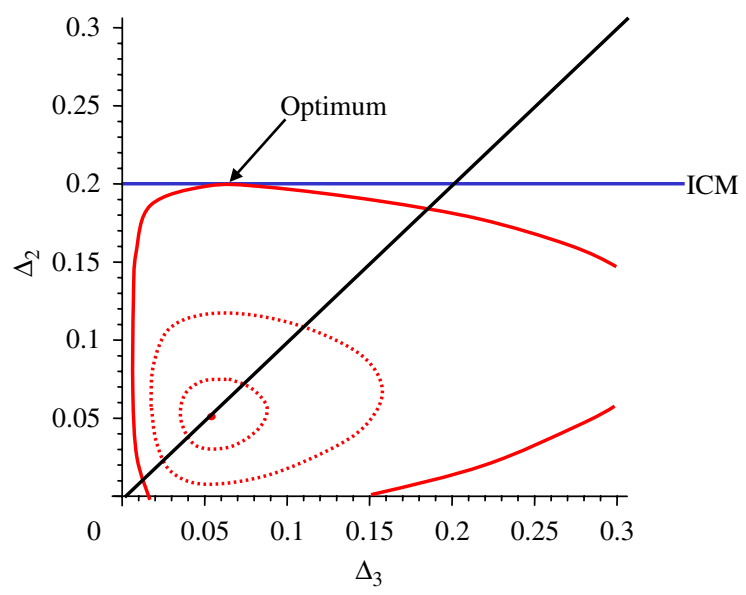

Fig. 8. Continuous search effort.

The intuition for our result is straightforward and builds on the intuition developed in the previous section. A binding ICM constraint implies that insurance to short-term unemployed must be more limited than what is required to induce the second-best amount of search effort in absence of the ICM constraint. This, however, does not imply that also the insurance for long-term unemployed must be reduced below what is required to induce the right amount of search effort.

\subsection{Loss of skills and long-term unemployment}

So far, we have considered the third state as an administrative state, used as a proxy for the unemployment duration of the agent. Unemployment was assumed to have no other effect than depleting the financial assets of the agent; hiring rates and search costs remained constant. However, it is easy to relax this assumption and analyze how the path of benefits should be constructed if the unemployment duration also has real direct effects on, e.g., search costs and hiring probabilities. ${ }^{23}$ Specifically, let $s_{2}$ and $s_{3}$ denote the search costs in states 2 and 3 and, correspondingly, $h_{2}$ and $h_{3}$ denote the state dependent hiring probabilities. The idea that the human capital of the unemployed depreciates during the unemployment spell (or that the individual "learns how to be unemployed") is captured by the assumption $h_{2}>h_{3}$ and/or $s_{2}<s_{3}$, implying $s_{2} / h_{2}<s_{3} / h_{3}$.

It is straightforward to show that the IC2 and IC3 constraints now become

$$
\begin{aligned}
& \Delta_{2} \geqslant-\gamma^{-1} \ln \left(1-\gamma r \frac{s_{2}}{h_{2}}\right), \\
& \Delta_{3} \geqslant-\gamma^{-1} \ln \left(1-\gamma r \frac{s_{3}}{h_{3}}\right),
\end{aligned}
$$

respectively, where $-\gamma^{-1} \ln \left(1-\gamma r s_{2} / h_{2}\right)<-\gamma^{-1} \ln \left(1-\gamma r s_{3} / h_{3}\right)$ so that the IC3 constraint crosses the IC2 condition below the $45^{\circ}$ line. If the binding constraints are IC2 and IC3

\footnotetext{
${ }^{23}$ Similarly, we could easily analyze the case when the prospective wage depends on unemployment duration.
} 
(small moving costs), we must then $\Delta_{3}>\Delta_{2}$. Using the implementation equations (using the different search costs and hiring rates), we find that in this case, the optimal benefit schedule should be downward sloping $\left(b_{2}>b_{3}\right)$. If the ICM constraint binds, rather than IC2, the possibility that the optimal benefit profile should be upward sloping remains.

\subsection{A menu of contracts}

Finally let us note that our model can also easily handle more complicated UI schemes, e.g., menus. ${ }^{24}$ In particular, let us consider the case when the insurer allows individuals losing their job to either get a lump-sum transfer $T$, or a possibly non-constant UI-benefit stream. ${ }^{25}$ Since the effective cost of moving is now $m-T$, the incentive constraint for individuals with the opportunity to move now becomes,

$$
\Delta_{2}=r(m-T),
$$

i.e., a positive $T$ slackens the constraint (moves it down in the figures). Increasing $T$ to a sufficiently large extent leads to a situation like that in Fig. 6, where IC2 and IC3 bind. Potentially, it is optimal to set $T=m$-full subsidization. This is the case if unemployed without moving opportunities prefer UI benefits over $T$, so that a separation between the groups is achieved also when the moving cost is fully insured. If such separation is not achieved under full insurance but should be in optimum, $T$ must be reduced so that unemployed individuals choose UI benefits. We note that we cannot increase the relative attractiveness of UI-benefits by raising the latter, since this would violate the IC2 and IC3 conditions, which continue to bind.

To analyze whether separation is achieved, we need to add another state to the analysis, namely to be unemployed without benefit, which makes a two-dimensional graphical analysis impractical. The analytical analysis remains simple, however. Setting the income of unemployed to zero, the consumption constant associated with being unemployed without benefits is given by

$$
\sigma_{u}=-s-\tau+\frac{h\left(1-\mathrm{e}^{-\gamma\left(\sigma_{1}-\sigma_{u}\right)}\right)}{\gamma r},
$$

so that $\sigma_{u}$ is a function of $\sigma_{1}$ only. The incentive constraint implying that unemployed do not choose the lump-sum transfer is then $\sigma_{2}-\sigma_{u} \geqslant r T$, and it is easily checked if this is satisfied in the equilibrium. If not, $T$ must be reduced. If the ICM condition is slack, benefits should be constant. However, as $T$ is reduced, the ICM condition might eventually bind, once more calling for an upward sloping benefit schedule.

\section{Conclusion}

In this paper, we have argued that there are reasons to believe that an important informational problem associated with unemployment insurance has been neglected in the previous literature. This problem stems from the fact that unemployed individuals

\footnotetext{
${ }^{24}$ Some UI schemes offer this type of menus; in particular, in the period of large unemployment (end of the 1980s and beginning of the 1990s) the Spanish Unemployment agency offered the option of a lump-sum transfer or standard UI payments.

${ }^{25}$ For simplicity, let us disregard the case of voluntary separations as discussed above.
} 
sometimes have the option of making an investment that could increase their chances of finding a job. Examples of such investments are retraining and moving to another location. Since it is reasonable to assume that it is difficult or impossible to observe who has these options, the UI system should give incentives for people to take advantage of any reasonable option to increase their labor market prospects. If such options arrive at a reasonably high rate or exist already at the onset of the unemployment spell, this can have important qualitative implications for how the UI system should be designed.

By deriving graphical and analytical closed-form solutions, we have shown how a simple UI system should be constructed to provide sufficient incentives to move or retrain without excessively reducing the insurance value of the unemployment benefits. Unless the hiring rates of long-term unemployed are very low and search costs too high, this requires an initial period of relatively low benefits. The intuition here is straightforward, by setting initial benefits at a low level, individuals with good opportunities to get new jobs are induced to exploit these and quickly leave the pool of unemployed. On the other hand, individuals with worse opportunities value insurance against long-term unemployment more than insurance against short-term unemployment. The value of the UI system can therefore be maintained by providing more generous benefits for long-term unemployment, calling for an upward sloping benefit profile.

We have assumed that individuals can self-insure via unobservable savings, i.e., that individual consumption is unobservable or, for some other reason, non-contractable. If, in contrast, the insurer has control over the consumption of the individual, it is well known that a downward sloping path of consumption (and benefits, if the individual has no other income) provides the best trade-off between good search incentives and insurance. In a working paper version of this paper (Hassler and Rodríguez Mora, 2003), we analyze the case when individuals have no access to a market for saving and borrowing. In this case, we show that it is optimal to have constant benefits if the moving constraint binds while search constraints are slack. The reason for this is that there is no point in punishing unsuccessful search by reducing consumption as the unemployment spell continues if the search constraints are slack anyhow.

With savings, the downward sloping consumption profile is achieved voluntarily as individuals deplete their assets. This is true in general but under CARA preferences, the downward slope of consumption that is optimal with search moral hazard is achieved with constant benefits. Under the perhaps more realistic assumption of constant relative riskaversion, the analysis is greatly complicated by the fact that search incentives would depend on asset holdings. Shimer and Werning(2007) show that the behavior of an unemployed individual with CRRA preferences is similar to that of an individual with CARA preference if they have the same riskaversion and access to a riskless bond. However, with CRRA preferences, the degree of riskaversion changes with individual asset holdings. Therefore, incentive compatibility would not in general be consistent with benefits that are independent of individual asset holdings. However, the intuition for the results in this paper does not appear to be related to such effects. In our model, the preference for increasing benefits arises from the need to separate between the two types of workers and the fact that individual assets are depleted during unemployment (which is true for general specifications of utility, in particular for CRRA, as shown in e.g., Hassler and Rodríguez Mora, 1999). Both mechanisms are likely to be present also under more general preference specifications. However, since search incentives in general depend on 
asset holdings and the duration of unemployment is likely to be correlated with the individual's asset holdings, unobservability of the latter may have consequences for optimal benefit time profiles. For example, if the search incentives are reinforced as wealth is depleted and individuals with long unemployment spells are likely to have less wealth, this might strengthen the case for increasing benefits. On the other hand, with wealth effects present, it could also be the case that individuals with opportunities to move do not do so until their assets are run down sufficiently. An initial period of low benefits may then not be sufficient to separate individuals who can move from those who cannot and upward sloping benefits could be suboptimal.

We have argued that under some circumstances, upward sloping benefits could be optimal, challenging the conventional wisdom that benefits should fall over the unemployment spell. We finally want to provide some word of caution. Neither the assumptions we have used nor the ones used to derive the conventional wisdom are perfectly realistic. The incentive problems operating during an unemployment spell are specific to the individual, time varying and wealth dependent. The moving costs is not a constant, but rather specific to the particular moving opportunity and finding a moving opportunity may require costly search, blurring the difference between the two types of incentive constraints analyzed in this paper. Furthermore, the market for borrowing and saving is neither perfect nor non-existent and CRRA is probably a better description of preferences than CARA, implying wealth effects on incentives. All this implies that incentive constraints are heterogeneous, partly determined by unobserved individual characteristics and state variables. Therefore, a quantitative analysis must recognize the possibility that some incentive constraints should optimally be violated. The social cost of this depends on the number of people for whom the constraint is violated. Finding the optimal benefit system then requires information on the distribution of the unobserved individual characteristics and how the evolution of partly endogenous state variables depend on the characteristics of the UI-system. This is left to future research.

\section{Acknowledgements}

The authors thank Christina Lönnblad for editorial assistance and Kjetil Storesletten, Jaume Ventura and Nicola Pavoni for valuable comments. JVRM acknowledges financial support from the Spanish Ministry of Education, project number SEJ2004-06877. JH thanks the Swedish Research Council for financial support.

\section{Appendix A}

\section{A.1. Bellman equations and consumption constants}

We start by conjecturing that the value function can be written $-\mathrm{e}^{-\gamma\left(r A_{t}+\sigma_{j}\right)}$ for the undetermined coefficients $\sigma_{j}, j \in\{1,2,3\}$. We also conjecture that consumption net of interest rates, i.e., $\sigma_{j}=c_{t, j}-r A_{t}$ is independent of assets. The proof then proceeds by showing that this consumption rule maximizes the Bellman equations and that the Bellman equations are satisfied for a unique set of $\sigma_{j}^{s}$. 
The Bellman equation of an employed individual is

$$
\begin{aligned}
-\frac{1}{r} \mathrm{e}^{-\gamma\left(r A_{t}+\sigma_{1}\right)}= & \max _{\sigma}-\mathrm{e}^{-\gamma\left(r A_{t}+\sigma\right)} \mathrm{d} t-(1-r \mathrm{~d} t)(1-q \mathrm{~d} t) \frac{1}{r} \mathrm{e}^{-\gamma\left(r A_{t+\mathrm{d} t}+\sigma_{1}\right)} \\
& -(1-r \mathrm{~d} t) q \mathrm{~d} t \frac{1}{r}\left[(1-p) \mathrm{e}^{-\gamma\left(r A_{t+\mathrm{d} t}+\sigma_{2}\right)}+p \mathrm{e}^{-\gamma\left(r A_{t+\mathrm{d} t}-m+\sigma_{2}\right)}\right] .
\end{aligned}
$$

Using the budget constraint, $A_{t+\mathrm{d} t}=A_{t}+r(w-\tau-\sigma) \mathrm{d} t$, and dividing by $\mathrm{e}^{-\gamma r A_{t}}$, this becomes

$$
\begin{aligned}
-\frac{1}{r} \mathrm{e}^{-\gamma \sigma_{1}}= & \max _{\sigma}-\mathrm{e}^{-\gamma \sigma} \mathrm{d} t-(1-r \mathrm{~d} t)(1-q \mathrm{~d} t) \frac{1}{r} \mathrm{e}^{-\gamma\left(r(w-\tau-\sigma) \mathrm{d} t+\sigma_{1}\right)} \\
& -(1-r \mathrm{~d} t) q \mathrm{~d} t \frac{1}{r}\left[(1-p) \mathrm{e}^{-\gamma\left(r(w-\tau-\sigma) \mathrm{d} t+\sigma_{2}\right)}+p \mathrm{e}^{-\gamma\left(r(w-\tau-\sigma) \mathrm{d} t-r m+\sigma_{1}\right)}\right] .
\end{aligned}
$$

Using the first-order linear approximation, $\mathrm{e}^{-\gamma\left(r(w-\tau-\sigma) \mathrm{d} t+\sigma_{1}\right)} \approx \mathrm{e}^{-\gamma \sigma_{1}}-\gamma r(w-\tau-\sigma)$ $\mathrm{d} t \mathrm{e}^{-\gamma \sigma_{1}}$, adding $(1 / r) \mathrm{e}^{-\gamma \sigma_{1}}$ to both sides, dividing by $\mathrm{d} t$ and letting $\mathrm{d} t$ approach zero, yields

$$
\begin{aligned}
0= & \max _{\sigma}\left\{-r \mathrm{e}^{-\gamma\left(\sigma-\sigma_{1}\right)}+r+\gamma r(w-\tau-\sigma)\right\} \\
& +q\left(1-(1-p) \mathrm{e}^{-\gamma\left(\sigma_{2}-\sigma_{1}\right)}-p \mathrm{e}^{\gamma r m}\right) .
\end{aligned}
$$

Similarly, for the short-term and long-run unemployed, we obtain

$$
\begin{aligned}
0= & \max _{\sigma}\left\{-r \mathrm{e}^{-\gamma\left(\sigma-\sigma_{2}\right)}+\gamma r\left(b_{2}-s-\tau-\sigma\right)\right\} \\
& +r+h+f-h \mathrm{e}^{-\gamma\left(\sigma_{1}-\sigma_{2}\right)}-f \mathrm{e}^{-\gamma\left(\sigma_{3}-\sigma_{2}\right)} \\
0= & \max _{\sigma}\left\{-r \mathrm{e}^{-\gamma\left(\sigma-\sigma_{3}\right)}+\gamma r\left(b_{3}-s-\tau-\sigma\right)\right\} \\
& +r+h\left(1-\mathrm{e}^{-\gamma\left(\sigma_{1}-\sigma_{3}\right)}\right) .
\end{aligned}
$$

Eqs. (20) and (21) are maximized at $\sigma=\sigma_{j}$, implying that these values maximize the RHS's of the Bellman equations.

Substituting $\sigma_{1}, \sigma_{2}, \sigma_{3}$, respectively, for $\sigma$ in (20), (21) and (22) solves the maxima. Finally, solving for gives the $\sigma_{j}^{s}$ gives (6), which by construction then solves the Bellman equations.

Taking the difference between lines 1 and 2 and between 1 and 3 in (6) and solving for $b_{2}$ and $b_{3}$, we obtain the implementation mapping (16).

\section{A.2. The IC2 and IC3 conditions}

We first note if a long-term unemployed does not search, she gets an income $b_{3}-\tau$ forever, implying a utility $-(1 / r) \mathrm{e}^{-\gamma r A_{t}} \mathrm{e}^{-\gamma\left(b_{3}-\tau\right)}$, while she gets $-(1 / r) \mathrm{e}^{-\gamma r A_{t}} \mathrm{e}^{-\gamma \sigma_{3}}$ if she searches. Therefore, we need $\sigma_{3} \geqslant b_{3}-\tau$ to induce search of the long-term unemployed. Using (6), this implies

$$
\Delta_{3} \geqslant-\gamma^{-1} \ln \left(1-\gamma r \frac{s}{h}\right)
$$

which is the IC3-condition.

For the short-term unemployed, we compute the value associated with a one-period deviation, i.e., no search in the current employment state, conditional on searching in 
future states. This value is $-\mathrm{e}^{-\gamma r A_{t}} \mathrm{e}^{-\gamma c_{2, n}} / r$, where $\sigma_{2, n}$ satisfies

$$
\sigma_{2, n}=b_{2}-\tau+\frac{f\left(1-\mathrm{e}^{-\gamma\left(\sigma_{3}-\sigma_{2, n}\right)}\right)}{\gamma r} .
$$

The IC2 constraint is given by

$$
\sigma_{2}-\sigma_{2, n} \geqslant 0 \text {. }
$$

Furthermore,

$$
\begin{aligned}
\sigma_{2}-\sigma_{2, n} & =\left(-s+\frac{h\left(1-\mathrm{e}^{-\gamma \Delta_{2}}\right)}{\gamma r}-\frac{f\left(\mathrm{e}^{\gamma\left(\Delta_{3}-\Delta_{2}\right)}-\mathrm{e}^{-\gamma\left(\sigma_{3}-\sigma_{2, n}\right)}\right)}{\gamma r}\right) \\
& =\left(-s+\frac{h}{\gamma r}\left(1-\mathrm{e}^{-\gamma \Delta_{2}}\right)-\frac{f}{\gamma r} \mathrm{e}^{\gamma\left(\Delta_{3}-\Delta_{2}\right)}\left(1-\mathrm{e}^{-\gamma\left(\sigma_{2}-\sigma_{2, n}\right)}\right)\right) \\
& \equiv R\left(\sigma_{2}-\sigma_{2, n}\right) .
\end{aligned}
$$

Clearly, $R$ is a monotonously decreasing function with a horizontal asymptote at $-s+$ $h / \gamma r\left(1-\mathrm{e}^{-\gamma \Delta_{2}}\right)-(f / \gamma r) \mathrm{e}^{\gamma\left(\Delta_{3}-\Delta_{2}\right)}$ (achieved as $\sigma_{2}-\sigma_{2, n}$ approaches infinity), approaches infinity as $\sigma_{2}-\sigma_{2, n}$ approaches minus infinity and $R(0)=-s+h / \gamma r\left(1-\mathrm{e}^{-\gamma \Delta_{2}}\right)$. The solution to (24) is the unique fixed-point of $R$. This value is non-negative if and only if $-s+h / \gamma r\left(1-\mathrm{e}^{-\gamma \Delta_{2}}\right) \geqslant 0$. So

$$
\sigma_{2} \geqslant \sigma_{2, n} \Leftrightarrow \Delta_{2} \geqslant-\gamma^{-1} \ln \left(1-\frac{\gamma r s}{h}\right) .
$$

\section{A.3. Proof that results extend to $n$ unemployment states}

Suppose we have $n$ states, then the consumption constants are

$$
\begin{aligned}
& \sigma_{1}=w-\tau-q \frac{p \mathrm{e}^{\gamma r m}+(1-p) \mathrm{e}^{\gamma \Delta_{2}}-1}{\gamma r}, \\
& \sigma_{2}=b_{2}-s-\tau+h \frac{1-\mathrm{e}^{-\gamma \Delta_{2}}}{\gamma r}-f \frac{\mathrm{e}^{\gamma\left(\Delta_{3}-\Delta_{2}\right)}-1}{\gamma r}, \\
& \sigma_{3}=b_{3}-s-\tau+h \frac{1-\mathrm{e}^{-\gamma \Delta_{3}}}{\gamma r}-f_{3} \frac{\mathrm{e}^{\gamma\left(\Delta_{4}-\Delta_{3}\right)}-1}{\gamma r}, \\
& \vdots \\
& \sigma_{n-1}=b_{n-1}-s-\tau+h \frac{1-\mathrm{e}^{-\gamma \Delta_{n-1}}}{\gamma r}-f_{n-1} \frac{\mathrm{e}^{\gamma\left(\Delta_{n}-\Delta_{n-1}\right)}-1}{\gamma r}, \\
& \sigma_{n}=b_{n}-s-\tau+h \frac{1-\mathrm{e}^{-\gamma \Delta_{n}}}{\gamma r} .
\end{aligned}
$$

Now, $\tau=\sum_{s=2}^{n} b_{s} \Pi_{s}$, and assume the ICM constraint to be binding, so $\Delta_{2}=r m$, implying that we should minimize taxes. Using the above, and $\Delta_{2}=r m$ we have

$$
\Delta_{2}=w-b_{2}+s-q \frac{\mathrm{e}^{\gamma r m}-1}{\gamma r}-h \frac{1-\mathrm{e}^{-\gamma r m}}{\gamma r}+f \frac{\mathrm{e}^{\gamma\left(\Delta_{3}-\Delta_{2}\right)}-1}{\gamma r},
$$




$$
\begin{aligned}
& \Delta_{3}=w-b_{3}+s-q \frac{\mathrm{e}^{\gamma r m}-1}{\gamma r}-h \frac{1-\mathrm{e}^{-\gamma \Delta_{3}}}{\gamma r}+f_{3} \frac{\mathrm{e}^{\gamma\left(\Delta_{4}-\Delta_{3}\right)}-1}{\gamma r}, \\
& \vdots \\
& \Delta_{n-1}=w-b_{n-1}+s-q \frac{\mathrm{e}^{\gamma r m}-1}{\gamma r}-h \frac{1-\mathrm{e}^{-\gamma \Delta_{n-1}}}{\gamma r}+f_{n-1} \frac{\mathrm{e}^{\gamma\left(\Delta_{n}-\Delta_{n-1}\right)}-1}{\gamma r}, \\
& \Delta_{n}=w-b_{n}+s-q \frac{\mathrm{e}^{\gamma r m}-1}{\gamma r}-h \frac{1-\mathrm{e}^{-\gamma\left(\Delta_{n}\right)}}{\gamma r}
\end{aligned}
$$

or

$$
\begin{aligned}
b_{2}= & w-\Delta_{2}+s-q \frac{\mathrm{e}^{\gamma r m}-1}{\gamma r}-h \frac{1-\mathrm{e}^{-\gamma r m}}{\gamma r}+f_{2} \frac{\mathrm{e}^{\gamma\left(\Delta_{3}-\Delta_{2}\right)}-1}{\gamma r}, \\
b_{3}= & w-\Delta_{3}+s-q \frac{\mathrm{e}^{\gamma r m}-1}{\gamma r}-h \frac{1-\mathrm{e}^{-\gamma \Delta_{3}}}{\gamma r}+f_{3} \frac{\mathrm{e}^{\gamma\left(\Delta_{4}-\Delta_{3}\right)}-1}{\gamma r}, \\
\vdots & \\
b_{n-1}= & w-\Delta_{n-1}+s-q \frac{\mathrm{e}^{\gamma r m}-1}{\gamma r}-h \frac{1-\mathrm{e}^{-\gamma \Delta_{n-1}}}{\gamma r}+f_{n-1} \frac{\mathrm{e}^{\gamma\left(\Delta_{n}-\Delta_{n-1}\right)}-1}{\gamma r}, \\
b_{n}= & w-\Delta_{n}+s-q \frac{\mathrm{e}^{\gamma r m}-1}{\gamma r}-h \frac{1-\mathrm{e}^{-\gamma\left(\Delta_{n}\right)}}{\gamma r}, \\
\tau= & \Pi_{2}\left(w-r m+s-q \frac{\mathrm{e}^{\gamma r m}-1}{\gamma r}-h \frac{1-\mathrm{e}^{-\gamma r m}}{\gamma r}+f \frac{\mathrm{e}^{\gamma\left(\Delta_{3}-r m\right)}-1}{\gamma r}\right) \\
& +\sum_{i=3}^{n-1} \Pi_{3}\left(w-\Delta_{i}+s-q \frac{\mathrm{e}^{\gamma r m}-1}{\gamma r}-h \frac{1-\mathrm{e}^{-\gamma \Delta_{i}}}{\gamma r}+f_{s} \frac{\mathrm{e}^{\gamma\left(\Delta_{i+1}-\Delta_{i}\right)}-1}{\gamma r}\right) \\
& +\Pi_{n}\left(w-\Delta_{n}+s-q \frac{\mathrm{e}^{\gamma r m}-1}{\gamma r}-h \frac{1-\mathrm{e}^{-\gamma \Delta_{n}}}{\gamma r}\right) \cdot
\end{aligned}
$$

Removing constants,

$$
\begin{aligned}
\tau= & \text { constant }+\Pi_{2}\left(f \frac{\mathrm{e}^{\gamma\left(\Delta_{3}-r m\right)}}{\gamma r}\right)+\sum_{i=3}^{n-1} \Pi_{i}\left(-\Delta_{i}+h \frac{\mathrm{e}^{-\gamma \Delta_{i}}}{\gamma r}+f_{s} \frac{\mathrm{e}^{\gamma\left(\Delta_{i+1}-\Delta_{i}\right)}}{\gamma r}\right) \\
& +\Pi_{n}\left(-\Delta_{n}+h \frac{\mathrm{e}^{-\gamma \Delta_{n}}}{\gamma r}\right) .
\end{aligned}
$$

\section{First-order conditions are}

$$
\begin{aligned}
& \Delta_{i \in\{3, n-1\}} ; \Pi_{i-1} \frac{f_{i-1}}{r} \mathrm{e}^{\gamma\left(\Delta_{i}-\Delta_{i-1}\right)}-\Pi_{i}\left(1+\frac{h}{r} \mathrm{e}^{-\gamma \Delta_{i-1}}+\frac{f_{i}}{r} \mathrm{e}^{\gamma\left(\Delta_{i}-\Delta_{i-1}\right)}\right)=0, \\
& \Delta_{n} ; \Pi_{n-1} \frac{f_{n-1}}{r} \mathrm{e}^{\gamma\left(\Delta_{n}-\Delta_{n-1}\right)}-\Pi_{n}\left(1+\frac{h}{r} \mathrm{e}^{-\gamma \Delta_{n}}\right)=0,
\end{aligned}
$$

where $\Delta_{2}=\mathrm{rm}$. 
Suppose that this is satisfied for $\Delta_{3}=\Delta_{4}=\cdots \Delta_{n}=\Delta$. Then,

$$
\begin{aligned}
& \mathrm{e}^{\gamma(\Delta-r m)}=\frac{r \Pi_{3}}{f_{2} \Pi_{2}}\left(1+\frac{h}{r} \mathrm{e}^{-\gamma \Delta}+\frac{f_{3}}{r}\right), \\
& \frac{f_{i-1}}{r}=\frac{\Pi_{i}}{\Pi_{i-1}}\left(1+\frac{h}{r} \mathrm{e}^{-\gamma \Delta}+\frac{f_{i}}{r}\right), \\
& \frac{f_{n-1}}{r}=\frac{\Pi_{n}}{\Pi_{n-1}}\left(1+\frac{h}{r} \mathrm{e}^{-\gamma \Delta}\right)
\end{aligned}
$$

or

$$
\begin{aligned}
\mathrm{e}^{\gamma(\Delta-r m)} & =\frac{r \Pi_{3}}{f_{2} \Pi_{2}}\left(1+\frac{h}{r} \mathrm{e}^{-\gamma \Delta}+\frac{\Pi_{4}}{\Pi_{3}}\left(1+\frac{h}{r} \mathrm{e}^{-\gamma \Delta}+\frac{f_{4}}{r}\right)\right) \\
& =\frac{r \Pi_{3}}{f_{2} \Pi_{2}}\left(1+\frac{h}{r} \mathrm{e}^{-\gamma \Delta}+\frac{\Pi_{4}}{\Pi_{3}}\left(1+\frac{h}{r} \mathrm{e}^{-\gamma \Delta}+\frac{\Pi_{5}}{\Pi_{4}}\left(1+\frac{h}{r} \mathrm{e}^{-\gamma \Delta}+\frac{f_{5}}{r}\right)\right)\right) \\
& =\frac{r}{f_{2}}\left(1+\frac{h}{r} \mathrm{e}^{-\gamma \Delta}\right)\left(\left(\frac{\Pi_{3}}{\Pi_{2}}+\frac{\Pi_{4}}{\Pi_{3}}+\cdots+\frac{\Pi_{n-1}}{\Pi_{n-2}}\right)+\frac{\Pi_{n}}{\Pi_{2}}\right) \\
& =\frac{r}{f_{2}}\left(1+\frac{h}{r} \mathrm{e}^{-\gamma \Delta}\right)\left(\sum_{i=3}^{n-1} \frac{\Pi_{i}}{\Pi_{i-1}}+\frac{\Pi_{n}}{\Pi_{2}}\right) .
\end{aligned}
$$

Clearly, there exists a $\Delta^{*}$ such that this is satisfied, consequently $\Delta_{i}=\Delta^{*} \forall i \in\{3,4, \ldots, n\}$ satisfies all first-order conditions. This allocation is then implemented by a $\tilde{b}_{2}^{*}$ and a constant benefit sequence $\tilde{b}_{3}^{*}=\tilde{b}_{4}^{*}=\cdots \tilde{b}_{n}^{*}$. Finally, we note that since individuals face identical conditions in states $3, \ldots, n$, the allocation would not change if the number of states were reduced as long as $n>3$. Thus, the optimal value of $b_{2}$ is independent of $n$ if $n>3$. Consequently, the optimal benefit schedule is to have $b_{2}=b_{2}^{*}$ and a constant benefit level $b_{3}=b_{3}^{*}$ thereafter.

\section{A.4. Derivation of (9)}

The consumption difference is

$$
\begin{aligned}
\Delta_{2} & =w-\tau-q \frac{p \mathrm{e}^{\gamma r m}+(1-p) \mathrm{e}^{\gamma\left(\Delta_{2}\right)}-1}{\gamma r}-\left(b_{2}-s-\tau+h \frac{1-\mathrm{e}^{-\gamma\left(\Delta_{2}\right)}}{\gamma r}\right) \\
& =w-b_{2}+s-q \frac{p \mathrm{e}^{\gamma r m}+(1-p) \mathrm{e}^{\gamma\left(\Delta_{2}\right)}-1}{\gamma r}-\left(h \frac{1-\mathrm{e}^{-\gamma\left(\Delta_{2}\right)}}{\gamma r}\right),
\end{aligned}
$$

giving

$$
b_{2}=w+s-q \frac{p \mathrm{e}^{\gamma r m}-1}{\gamma r}-\frac{h}{\gamma r}-\Delta_{2}-q \frac{(1-p) \mathrm{e}^{\gamma \Delta_{2}}}{\gamma r}+\frac{h \mathrm{e}^{-\gamma \Delta_{2}}}{\gamma r} .
$$

Collecting constants we get

$$
\sigma_{1}=w-\Pi_{2}\left(w+s-q \frac{p \mathrm{e}^{\gamma r m}-1}{\gamma r}-\frac{h}{\gamma r}-\Delta_{2}-q \frac{(1-p) \mathrm{e}^{\gamma \Delta_{2}}}{\gamma r}+\frac{h \mathrm{e}^{-\gamma \Delta_{2}}}{\gamma r}\right)
$$




$$
\begin{aligned}
& -q \frac{p \mathrm{e}^{\gamma r m}-1}{\gamma r}-q \frac{(1-p) \mathrm{e}^{\gamma \Delta_{2}}}{\gamma r} \\
= & w-\Pi_{2}\left(w+s-q \frac{p \mathrm{e}^{\gamma r m}-1}{\gamma r}-\frac{h}{\gamma r}\right)-q \frac{p \mathrm{e}^{\gamma r m}-1}{\gamma r} \\
& +\Pi_{2}\left(\Delta_{2}+q \frac{(1-p) \mathrm{e}^{\gamma \Delta_{2}}}{\gamma r}-\frac{h \mathrm{e}^{-\gamma \Delta_{2}}}{\gamma r}\right)-q \frac{(1-p) \mathrm{e}^{\gamma \Delta_{2}}}{\gamma r} \\
= & \kappa+\Pi_{2}\left(\Delta_{2}+q \frac{(1-p) \mathrm{e}^{\gamma \Delta_{2}}}{\gamma r}-\frac{h \mathrm{e}^{-\gamma \Delta_{2}}}{\gamma r}\right)-q \frac{(1-p) \mathrm{e}^{\gamma \Delta_{2}}}{\gamma r} \\
= & \kappa+\Pi_{2}\left(\Delta_{2}-\frac{h \mathrm{e}^{-\gamma \Delta_{2}}}{\gamma r}\right)-\left(1-\Pi_{2}\right) q \frac{(1-p) \mathrm{e}^{\gamma \Delta_{2}}}{\gamma r} .
\end{aligned}
$$

\section{A.5. Derivation of 14}

Doing the substitution in the text and collecting endogenous terms, we have

$$
\begin{aligned}
\sigma_{1}= & w-\Pi_{2}\left(w+s-q \frac{p \mathrm{e}^{\gamma r m}-1}{\gamma r}-(h+f) \frac{1}{\gamma r}\right)-\Pi_{3}\left(w+s-q \frac{p \mathrm{e}^{\gamma r m}-1}{\gamma r}-\frac{h}{\gamma r}\right) \\
& -q \frac{p \mathrm{e}^{\gamma r m}-1}{\gamma r}-\Pi_{2}\left(-\Delta_{2}-q \frac{(1-p) \mathrm{e}^{\gamma \Delta_{2}}}{\gamma r}+h \frac{\mathrm{e}^{-\gamma \Delta_{2}}}{\gamma r}+f \frac{\mathrm{e}^{\gamma\left(\Delta_{3}-\Delta_{2}\right)}}{\gamma r}\right) \\
& -\Pi_{3}\left(-\Delta_{3}-q \frac{(1-p) \mathrm{e}^{\gamma \Delta_{2}}}{\gamma r}+h \frac{\mathrm{e}^{-\gamma \Delta_{3}}}{\gamma r}\right)-q \frac{(1-p) \mathrm{e}^{\gamma \Delta_{2}}}{\gamma r} \\
= & \kappa_{2}+\Pi_{2} \Delta_{2}+\Pi_{3} \Delta_{3}-\left(1-\Pi_{2}-\Pi_{3}\right) q \frac{(1-p) \mathrm{e}^{\gamma \Delta_{2}}}{\gamma r} \\
& -\Pi_{2}\left(h \frac{\mathrm{e}^{-\gamma \Delta_{2}}}{\gamma r}+f \frac{\mathrm{e}^{\gamma\left(\Delta_{3}-\Delta_{2}\right)}}{\gamma r}\right)-\Pi_{3} h \frac{\mathrm{e}^{-\gamma \Delta_{3}}}{\gamma r} .
\end{aligned}
$$

\section{A.6. Indifference curves}

The objective function is

$$
\begin{aligned}
\sigma_{1}= & \kappa_{2}+\Pi_{2} \Delta_{2}+\Pi_{3} \Delta_{3}-\left(1-\Pi_{2}-\Pi_{3}\right) \frac{q(1-p)}{\gamma r} \mathrm{e}^{\gamma \Delta_{2}} \\
& -\Pi_{2}\left(h \frac{\mathrm{e}^{-\gamma \Delta_{2}}}{\gamma r}+f \frac{\mathrm{e}^{\gamma\left(\Delta_{3}-\Delta_{2}\right)}}{\gamma r}\right)-\Pi_{3} h \frac{\mathrm{e}^{-\gamma \Delta_{3}}}{\gamma r} .
\end{aligned}
$$

Differentiation gives

$$
\begin{gathered}
\left(\Pi_{2}-\left(1-\Pi_{2}-\Pi_{3}\right) \frac{q(1-p)}{r} \mathrm{e}^{\gamma \Delta_{2}}+\Pi_{2} h \frac{\mathrm{e}^{-\gamma \Delta_{2}}}{r}+\Pi_{2} f \frac{\mathrm{e}^{\gamma\left(\Delta_{3}-\Delta_{2}\right)}}{r}\right) \mathrm{d} \Delta_{2} \\
=-\left(\Pi_{3}-\Pi_{2} f \frac{\mathrm{e}^{\gamma\left(\Delta_{3}-\Delta_{2}\right)}}{r}+\Pi_{3} h \frac{\mathrm{e}^{-\gamma \Delta_{3}}}{r}\right) \mathrm{d} \Delta_{3}
\end{gathered}
$$




$$
\begin{aligned}
\left.\frac{\mathrm{d} \Delta_{2}}{\mathrm{~d} \Delta_{3}}\right|_{\sigma_{1}} \text { constant } & =-\frac{\left(\Pi_{3}-\Pi_{2} f \frac{\mathrm{e}^{\gamma\left(\Delta_{3}-\Delta_{2}\right)}}{r}+\Pi_{3} h \frac{\mathrm{e}^{-\gamma \Delta_{3}}}{r}\right)}{\left(\Pi_{2}-\left(1-\Pi_{2}-\Pi_{3}\right) \frac{q(1-p)}{r} \mathrm{e}^{\gamma \Delta_{2}}+\Pi_{2} h \frac{\mathrm{e}^{-\gamma \Delta_{2}}}{r}+\Pi_{2} f \frac{\mathrm{e}^{\gamma\left(\Delta_{3}-\Delta_{2}\right)}}{r}\right)} \\
& =-\frac{\frac{f}{h+r}-\frac{1}{r}\left(f \mathrm{e}^{\gamma\left(\Delta_{3}-\Delta_{2}\right)}-\frac{f h}{h+r} \mathrm{e}^{-\gamma \Delta_{3}}\right)}{1-\frac{1}{r}\left((r+h+f) \mathrm{e}^{\gamma \Delta_{2}}-h \mathrm{e}^{-\gamma \Delta_{2}}-f \mathrm{e}^{\gamma\left(\Delta_{3}-\Delta_{2}\right)}\right)} .
\end{aligned}
$$

\section{A.7. Different search and hiring probabilities}

Here, we formally analyze the case when $s$ and $h$ are state dependent. We first have that

$$
\begin{aligned}
& \sigma_{1}=w-\tau-q \frac{p \mathrm{e}^{\gamma r m}+(1-p) \mathrm{e}^{\gamma\left(\Delta_{2}\right)}-1}{\gamma r} \\
& \sigma_{2}=b_{2}-s_{2}-\tau+h_{2} \frac{1-\mathrm{e}^{-\gamma\left(\Delta_{2}\right)}}{\gamma r}-f \frac{\mathrm{e}^{\gamma\left(\Delta_{3}-\Delta_{2}\right)}-1}{\gamma r} \\
& \sigma_{3}=b_{3}-s_{3}-\tau+h_{3} \frac{1-\mathrm{e}^{-\gamma\left(\Delta_{3}\right)}}{\gamma r}
\end{aligned}
$$

The IC2 and IC3 conditions are

$$
\begin{aligned}
& \Delta_{2} \geqslant-\gamma^{-1} \ln \left(1-\gamma r \frac{s_{2}}{h_{2}}\right), \\
& \Delta_{3} \geqslant-\gamma^{-1} \ln \left(1-\gamma r \frac{s_{3}}{h_{3}}\right)
\end{aligned}
$$

and the implementation equations

$$
\begin{aligned}
& b_{2}=w+s_{2}-\Delta_{2}-\frac{q\left(\mathrm{e}^{\gamma \Delta_{2}}-1\right)+h_{2}\left(1-\mathrm{e}^{-\gamma \Delta_{2}}\right)-f\left(\mathrm{e}^{\gamma\left(\Delta_{3}-\Delta_{2}\right)}-1\right)}{\gamma r}, \\
& b_{3}=w+s_{3}-\Delta_{3}-\frac{q\left(\mathrm{e}^{\gamma \Delta_{2}}-1\right)+h_{3}\left(1-\mathrm{e}^{-\gamma \Delta_{3}}\right)}{\gamma r} .
\end{aligned}
$$

Fixing $\Delta_{2}$ and assuming that $s_{3}$ increases while respecting $\Delta_{3}=-\gamma^{-1} \ln \left(1-\gamma r s_{3} / h_{3}\right)$, we see that

$$
\begin{aligned}
\frac{\mathrm{d} b_{2}}{\mathrm{~d} s_{3}} & =\frac{\partial b_{2}}{\partial \Delta_{3}} \frac{\partial \Delta_{3}}{\partial s_{3}}=\frac{f \mathrm{e}^{\gamma\left(\Delta_{3}-\Delta_{2}\right)}}{r} \frac{r}{h_{3}-\gamma r s_{3}}=\frac{f \mathrm{e}^{\gamma\left(\Delta_{3}-\Delta_{2}\right)}}{h_{3}\left(1-\gamma r \frac{s_{3}}{h_{3}}\right)}>0 \\
\frac{\mathrm{d} b_{3}}{\mathrm{~d} s_{3}} & =1-\frac{\partial \Delta_{3}}{\partial s_{3}}\left(1+\frac{h_{3} \mathrm{e}^{-\gamma \Delta_{3}}}{r}\right) \\
& =1-\frac{r}{h_{3}-\gamma r s_{3}} \frac{r+h_{3} \mathrm{e}^{-\gamma \Delta_{3}}}{r}
\end{aligned}
$$




$$
\begin{aligned}
& =1-\frac{r}{h_{3}-\gamma r s_{3}} \frac{r+h_{3} \frac{h_{3}-\gamma r s_{3}}{h_{3}}}{r} \\
& =-\frac{r}{h_{3}\left(1-\gamma r \frac{s_{3}}{h_{3}}\right)}<0 .
\end{aligned}
$$

Similarly,

$$
\begin{aligned}
\frac{\mathrm{d} b_{2}}{\mathrm{~d} h_{3}} & =\frac{\partial b_{2}}{\partial \Delta_{3}} \frac{\partial \Delta_{3}}{\partial h_{3}}=\frac{f \mathrm{e}^{\gamma\left(\Delta_{3}-\Delta_{2}\right)}}{r} \frac{-r s_{3}}{h_{3}^{2}\left(1-\gamma r \frac{s_{3}}{h_{3}}\right)}<0 . \\
\frac{\mathrm{d} b_{3}}{\mathrm{~d} h_{3}} & =-\frac{\partial \Delta_{3}}{\partial h_{3}}\left(1+\frac{h_{3} \mathrm{e}^{-\gamma \Delta_{3}}}{r}\right)-\frac{\left(1-\mathrm{e}^{-\gamma \Delta_{3}}\right)}{\gamma r} \\
& =\frac{r s_{3}}{h_{3}\left(h_{3}-\gamma r s_{3}\right)} \frac{r+h_{3} \mathrm{e}^{\ln \left(1-\gamma r \frac{s_{3}}{h_{3}}\right)}}{r}-\frac{\left(1-\mathrm{e}^{\ln \left(1-\gamma r r_{3}\right)}\right)}{\gamma r} \\
& =\frac{r s_{3}}{h_{3}^{2}\left(1-\gamma r \frac{s_{3}}{h_{3}}\right)}>0 .
\end{aligned}
$$

A.8. The search constraints binds in the case of finite arrival rates of moving opportunities

Substituting $\Delta_{S, 2}=\Delta_{S, 3}=-\ln \left(1-\gamma r \frac{s}{h}\right) / \gamma$ in (19) yields

$$
\begin{aligned}
& \sigma_{1}=w-\tau-q \frac{\left(p \mathrm{e}^{\gamma \Delta_{M, 2}}+(1-p) \mathrm{e}^{-\ln \left(1-\gamma r r_{h}^{S}\right)}-1\right)}{\gamma r}, \\
& \sigma_{S, 2}=b_{2}-s-\tau+\frac{h\left(1-\mathrm{e}^{\ln \left(1-\gamma r r_{h}^{S}\right)}\right)}{\gamma r}, \\
& \sigma_{M, 2}=b_{2}-s-\tau+\mu \frac{\left(1-\mathrm{e}^{-\gamma\left(\Delta_{M, 2}-r m\right)}\right)}{\gamma r}+\frac{h\left(1-\mathrm{e}^{-\gamma \Delta_{M, 2}}\right)}{\gamma r} .
\end{aligned}
$$

Furthermore, setting the difference between lines 1 and 2 to $-\ln \left(1-\gamma r \frac{s}{h}\right) / \gamma$ and the difference between lines 1 and 3 to $\gamma \Delta_{M, 2}$ gives

$$
\begin{aligned}
& -\frac{\ln \left(1-\gamma r \frac{s}{h}\right)}{\gamma}=w-b_{2}-q \frac{\left(p \mathrm{e}^{\gamma \Delta_{M, 2}}+\frac{(1-p) h}{h-\gamma r s}-1\right)}{\gamma r} \\
& \Delta_{M, 2}=\left(w-\left(b_{2}-s\right)-\frac{q\left(p \mathrm{e}^{\gamma \Delta_{M, 2}}+\frac{(1-p) h}{h-\gamma r s}-1\right)+\mu\left(1-\mathrm{e}^{-\gamma\left(\Delta_{M, 2}-r m\right)}\right)+h\left(1-\mathrm{e}^{-\gamma \Delta_{M, 2}}\right)}{\gamma r}\right)
\end{aligned}
$$

which we need to solve numerically to get the two undetermined values $\Delta_{M, 2}$ and $b_{2}$.

\section{A.9. Calculation of $\Pi_{2}$ and $\Pi_{3}$}

To analyze the budget constraint we recall that we defined $\mu_{2, t}$ and $\mu_{3, t}$ as the mass of short-term and long-term unemployed, respectively. The law-of-motion for these variables 
(when the ICM constraint is satisfied) is

$$
\begin{aligned}
& \mu_{2, t+\mathrm{d} t}=(1-p) q \mathrm{~d} t\left(1-\mu_{2, t}-\mu_{3, t}\right)+\left(1-h_{2} \mathrm{~d} t-f \mathrm{~d} t\right) \mu_{2, t} \\
& \mu_{3, t+\mathrm{d} t}=f \mathrm{~d} t \mu_{2, t}+\left(1-h_{3} \mathrm{~d} t\right) \mu_{3, t}
\end{aligned}
$$

or

$$
\begin{aligned}
& \frac{\mu_{2, t+\mathrm{d} t}-\mu_{2, t}}{\mathrm{~d} t}=-(1-p) q \mu_{2, t}-(1-p) q \mu_{3, t}-\left(h_{2}+f\right) \mu_{2, t}+(1-p) q, \\
& \frac{\mu_{3, t+\mathrm{d} t}-\mu_{3, t}}{\mathrm{~d} t}=f \mu_{2, t}-h_{3} \mu_{3, t}
\end{aligned}
$$

taking the limit as $\mathrm{d} t \rightarrow 0$ yields

$$
\left[\begin{array}{l}
\dot{\mu}_{2, t} \\
\dot{\mu}_{2, t}
\end{array}\right]=\left[\begin{array}{ll}
-\left((1-p) q+h_{2}+f\right) & -(1-p) q \\
f & -h_{3}
\end{array}\right]\left[\begin{array}{l}
\mu_{2, t} \\
\mu_{3, t}
\end{array}\right]+\left[\begin{array}{l}
(1-p) q \\
0
\end{array}\right],
$$

with roots

$$
\rho_{1,2}=-\frac{F \pm \sqrt{\left(f+q(1-p)+h_{2}+h_{3}\right)^{2}-4\left(q\left(f+h_{3}\right)(1-p)+h_{3}\left(f+h_{2}\right)\right)}}{2},
$$

where $F \equiv\left(f+q(1-p)+h_{3}+h_{2}\right)$ and eigenvectors:

$$
\left\{\left[\begin{array}{l}
\frac{\rho_{1}+h_{3}}{f} \\
1
\end{array}\right]\right\} \leftrightarrow \rho_{1}, \quad\left\{\left[\begin{array}{c}
\frac{\rho_{2}+h_{3}}{f} \\
1
\end{array}\right]\right\} \leftrightarrow \rho_{2} .
$$

The steady state is

$$
\begin{aligned}
{\left[\begin{array}{l}
\bar{\mu}_{2} \\
\bar{\mu}_{3}
\end{array}\right] } & =-\left[\begin{array}{ll}
-\left((1-p) q+h_{2}+f\right) & -(1-p) q \\
f & -h_{3}
\end{array}\right]^{-1}\left[\begin{array}{l}
(1-p) q \\
0
\end{array}\right] \\
& =\left[\begin{array}{c}
\frac{h_{3}(1-p) q}{q(1-p)\left(h_{3}+f\right)+\left(h_{2}+f\right) h_{3}} \\
\frac{f(1-p) q}{q(1-p)\left(h_{3}+f\right)+\left(h_{2}+f\right) h_{3}}
\end{array}\right] .
\end{aligned}
$$

The solution to the system is then

$$
\left[\begin{array}{l}
\mu_{2, t} \\
\mu_{3, t}
\end{array}\right]=\left[\begin{array}{cc}
\frac{\rho_{1}+h_{3}}{f} & \frac{\rho_{2}+h_{3}}{f} \\
1 & 1
\end{array}\right]\left[\begin{array}{l}
c_{1} \mathrm{e}^{\rho_{1} t} \\
c_{2} \mathrm{e}^{\rho_{2} t}
\end{array}\right]+\left[\begin{array}{l}
\bar{\mu}_{2} \\
\bar{\mu}_{3}
\end{array}\right]
$$


Solving for the ex ante case when individuals are born employed $\left(\mu_{2,0}=\mu_{3,0}=0\right)$ yields

$$
\begin{aligned}
{\left[\begin{array}{l}
0 \\
0
\end{array}\right] } & =\left[\begin{array}{cc}
\frac{\rho_{1}+h_{3}}{f} & \frac{\rho_{2}+h_{3}}{f} \\
1 & 1
\end{array}\right]\left[\begin{array}{l}
c_{1} \\
c_{2}
\end{array}\right]+\left[\begin{array}{l}
\bar{\mu}_{2} \\
\bar{\mu}_{3}
\end{array}\right], \\
& \Rightarrow\left[\begin{array}{l}
c_{1} \\
c_{2}
\end{array}\right]=-\left[\begin{array}{ll}
\frac{\rho_{1}+h_{3}}{f} & \frac{\rho_{2}+h_{3}}{f} \\
1 & 1
\end{array}\right]^{-1}\left[\begin{array}{l}
\bar{\mu}_{2} \\
\bar{\mu}_{3}
\end{array}\right]=\left[\begin{array}{l}
\frac{\left(\rho_{2}+h_{3}\right) \mu_{3}-f \bar{\mu}_{2}}{\rho_{1}-\rho_{2}} \\
\frac{f \bar{\mu}_{2}-\left(h_{3}+\rho_{1}\right) \mu_{3}}{\rho_{1}-\rho_{2}}
\end{array}\right] .
\end{aligned}
$$

Thus, the complete solution is

$$
\begin{aligned}
& {\left[\begin{array}{l}
\mu_{2, t} \\
\mu_{3, t}
\end{array}\right]=} {\left[\begin{array}{ll}
\frac{\rho_{1}+h_{3}}{f} & \frac{\rho_{2}+h_{3}}{f} \\
1 & 1
\end{array}\right]\left[\begin{array}{l}
\frac{\left(\rho_{2}+h_{3}\right) \mu_{3}-f \bar{\mu}_{2}}{\rho_{1}-\rho_{2}} \mathrm{e}^{\rho_{1} t} \\
\frac{f \bar{\mu}_{2}-\left(h_{3}+\rho_{1}\right) \mu_{3}}{\rho_{1}-\rho_{2}} \mathrm{e}^{\rho_{2} t}
\end{array}\right] } \\
&+\left[\begin{array}{l}
\bar{\mu}_{2} \\
\bar{\mu}_{3}
\end{array}\right], \\
& \mu_{2, t}= \frac{\rho_{1}+h_{3}}{f} \frac{\bar{\mu}_{3}\left(\rho_{2}+h_{3}\right)-f \bar{\mu}_{2}}{\rho_{1}-\rho_{2}} \mathrm{e}^{\rho_{1} t} \\
&+\frac{\rho_{2}+h_{3} f \bar{\mu}_{2}-\bar{\mu}_{3}\left(h_{3}+\rho_{1}\right)}{f} \mathrm{e}^{\rho_{2} t}+\bar{\mu}_{2} \\
& \rho_{1}-\rho_{2} \\
& \mu_{3, t=} \frac{\bar{\mu}_{3}\left(\rho_{2}+h_{3}\right)-f \bar{\mu}_{2}}{\rho_{1}-\rho_{2}} \mathrm{e}^{\rho_{1} t} \\
&+\frac{f \bar{\mu}_{2}-\bar{\mu}_{3}\left(\rho_{1}+h_{3}\right)}{\rho_{1}-\rho_{2}} \mathrm{e}^{\rho_{2} t}+\bar{\mu}_{3} .
\end{aligned}
$$

We can now calculate $\Pi_{2}$ and $\Pi_{3}$ from

$$
\begin{aligned}
\Pi_{2}= & r \int_{0}^{\infty} \mathrm{e}^{-r t} \mu_{2, t} \mathrm{~d} t \\
= & r \int_{0}^{\infty} \frac{\rho_{1}+h_{3}}{f} \frac{\bar{\mu}_{3}\left(\rho_{2}+h_{3}\right)-f \bar{\mu}_{2}}{\rho_{1}-\rho_{2}} \mathrm{e}^{\left(\rho_{1}-r\right) t} \\
& +r \int_{0}^{\infty} \frac{\rho_{2}+h_{3} f \frac{f \bar{\mu}_{2}-\bar{\mu}_{3}\left(h_{3}+\rho_{1}\right)}{f} \mathrm{e}^{\left(\rho_{2}-r\right) t}}{\rho_{1}-\rho_{2}} \\
& +r \int_{0}^{\infty} \bar{\mu}_{2} \mathrm{e}^{-r t} \mathrm{~d} t \\
= & \frac{\left(h_{3}+r\right)(1-p) q \rho_{2} \rho_{1}}{\left(r-\rho_{2}\right)\left(r-\rho_{1}\right)\left((1-p) q\left(f+h_{3}\right)+h_{3}\left(f+h_{2}\right)\right)}
\end{aligned}
$$


and similarly

$$
\begin{aligned}
\Pi_{3} & =r \int_{0}^{\infty} \mathrm{e}^{-r t} \mu_{3, t} \mathrm{~d} t \\
& =\frac{f(1-p) q \rho_{2} \rho_{1}}{\left(r-\rho_{2}\right)\left(r-\rho_{1}\right)\left((1-p) q\left(f+h_{3}\right)+h_{3}\left(f+h_{2}\right)\right)} \\
& =\Pi_{2} \frac{f}{h_{3}+r} .
\end{aligned}
$$

\section{References}

Arpad, A., Pavoni, N., 2005. The efficient allocation of consumption under moral hazard and hidden access to the credit market. Journal of the European Economic Association 3, 370-381.

Baily, M.N., 1978. Some aspects of optimal unemployment insurance. Journal of Public Economics 10 (3), 379-402.

Bartel, A.P., 1979. The migration decision: What role does job mobility play? American Economic Review 69 (5), 775-786.

Cahuc, P., Lehmann, E., 2000. Should unemployment benefits decrease with the unemployment spell? Journal of Public Economics 77 (1), 135-153.

Chetty, R., 2006. A general formula for the optimal level of social insurance. Journal of Public Economics 90 (10-11), 1879-1901.

Chetty, R., Szeidl, A., 2007. Consumption commitments and risk preferences. Quarterly Journal of Economics $122(2), 831-877$.

Cullen, J.B., Gruber, J., 2000. Does unemployment insurance crowd out spousal labor supply? Journal of Labor Economics 18 (3), 546-572.

DaVanzo, J., 1978. Does unemployment affect migration?- evidence from micro data. Review of Economics and Statistics 6 (4), 504-514.

David Card, R.C., Weber, A., 2007. Cash-on-hand and competing models of intertemporal behavior: New evidence from the labor market. Quarterly Journal of Economics.

Engen, E.M., Gruber, J., 2001. Unemployment insurance and precautionary saving. Journal of Monetary Economics 47 (3), 545-579.

Gruber, J., 1997. The consumption smoothing effect of unemployment insurance. American Economic Review 87 (1), 192-205.

Hagedorn, M., Kaul, A., Memmel, T., 2003. An Adverse Selection Model of Optimal Unemployment Insurance. Mimeo, University Pompeu Fabra.

Hassler, J., Rodríguez Mora, J.V., 1999. Employment turnover and the public allocation of unemployment insurance. Journal of Public Economics 73 (1), 55-83.

Hassler, J., Rodríguez Mora, J.V., 2003. Should UI Benefits Really Fall Over Time? Mimeo, IIES, Stockholm University.

Hassler, J., Rodríguez Mora, J.V., Storesletten, K., Zilibotti, F., 2005. A positive theory of geographic mobility and social insurance. International Economic Review 46 (1), 263-303.

Holmlund, B., 1998. Unemployment insurance in theory and practice. Scandinavian Journal of Economics 100 (1), 113-141.

Hopenhayn, H.A., Nicolini, J.P., 1997. Optimal unemployment insurance. Journal of Political Economy 105 (2), 412-438.

McCormick, B., 1997. Regional unemployment and labour mobility in the uk. European Economic Review 41 (3-5), 581-589.

Pavoni, N., 2006. On optimal unemployment compensation. Journal of Monetary Economics, in press, doi:10.1016/j.jmoneco.2006.06.006.

Pissarides, C.A., Wadsworth, J., 1989. Unemployment and the inter-regional mobility of labour. Economic Journal 99 (397), 739-755.

Shavell, S., Weiss, L., 1979. The optimal payment of unemployment insurance benefits over time. Journal of Political Economy 87 (6), 1347-1362. 
Shimer, R., Werning, I., 2005. Liquidity and insurance for the unemployed. NBER Working Paper No. 11689. Shimer, R., Werning, I., 2007. Reservation wages and unemployment insurance. Quarterly Journal of Economics 122 (3), 1145-1185.

Wang, C., Williamson, S.D., 1996. Unemployment insurance with moral hazard in a dynamic economy. Carnegie-Rochester Conference Series on Public Policy 44, 1-41.

Werning, I., 2002. Optimal Unemployment Insurance with Unobservable Savings. Mimeo, University of Chicago. Wright, R., 1986. The redistributive roles of unemployment insurance and the dynamics of voting. Journal of Public Economics 31 (3), 377-399. 